\title{
Entanglement and $U(D)$-spin squeezing in symmetric multi-quDit systems and applications to quantum phase transitions in Lipkin-Meshkov-Glick D-level atom models
}

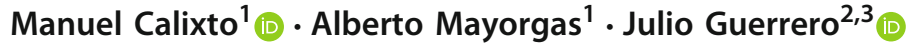

Received: 23 April 2021 / Accepted: 11 August 2021 / Published online: 13 September 2021

(c) The Author(s) 2021

\begin{abstract}
Collective spin operators for symmetric multi-quDit (namely identical $D$-level atom) systems generate a $\mathrm{U}(D)$ symmetry. We explore generalizations to arbitrary $D$ of SU(2)-spin coherent states and their adaptation to parity (multi-component Schrödinger cats), together with multi-mode extensions of NOON states. We write level, one- and two-quDit reduced density matrices of symmetric $N$-quDit states, expressed in the last two cases in terms of collective $\mathrm{U}(D)$-spin operator expectation values. Then, we evaluate level and particle entanglement for symmetric multi-quDit states with linear and von Neumann entropies of the corresponding reduced density matrices. In particular, we analyze the numerical and variational ground state of Lipkin-Meshkov-Glick models of 3-level identical atoms. We also propose an extension of the concept of SU(2)-spin squeezing to $\mathrm{SU}(D)$ and relate it to pairwise $D$-level atom entanglement. Squeezing parameters and entanglement entropies are good markers that characterize the different quantum phases, and their corresponding critical points, that take place in these interacting $D$-level atom models.
\end{abstract}

Keywords Quantum phase transitions · Many-body systems · Symmetric quDits · Coherent states $\cdot$ Parity $\cdot$ Pairwise entanglement $\cdot$ Spin squeezing

$凶 \quad$ Manuel Calixto

calixto@ugr.es

Alberto Mayorgas

albmayrey97@gmail.com

Julio Guerrero

jguerrer@ujaen.es

1 Department of Applied Mathematics and Institute Carlos I of Theoretical and Computational Physics (iC1), University of Granada, Fuentenueva s/n, 18071 Granada, Spain

2 Department of Mathematics, University of Jaen, Campus Las Lagunillas s/n, 23071 Jaen, Spain

3 Institute Carlos I of Theoretical and Computational Physics (iC1), University of Granada, Fuentenueva s/n, 18071 Granada, Spain 


\section{Introduction}

The development of quantum technologies partially relies on the efficient preparation of nonclassical atomic states and the exploitation of many-body entanglement [1-3] and spin squeezing [4], specially to enhance the sensitivity of precision measurements like in quantum metrology. Such is the case of many-body entangled (and spin-squeezed) states of cold atoms generated for instance in atom-atom collisions in Bose-Einstein condensates (BECs) [2].

Indistinguishable particles are naturally correlated due to exchange symmetry and there has been a long-standing debate on whether identical particle entanglement is physical or merely a mathematical artifact (see, e.g., [5] and references therein). Recent work like [6] shows indeed entanglement between identical particles as a consistent quantum resource in some typical optical and cold atomic systems with immediate practical impact. It can also be extracted and used as a resource for standard quantum information tasks [7]. Moreover, multi-partite entanglement of symmetric multi-qubit systems can add robustness and stability against the loss of a small number of particles [5].

Understanding the role of the indistinguishableness of identical bosons and quantum entanglement has been the subject of many recent work (see, e.g., [8,9] and references therein). We know that, for $N=2$ particles, any quantum state is either separable or entangled. However, for $N>2$, one needs further classifications for multi-partite entanglement [10]. Many different measurements have been proposed to detect and quantify quantum correlations [3]. We shall restrict ourselves to bipartite entanglement of pure states, where necessary conditions for separability in arbitrary dimensions exist.

In order to quantify entanglement between identical particles we shall follow Wang and Mølmer's [11] procedure, who wrote the reduced density matrix (RDM) of oneand two- qubits, extracted at random from a symmetric multi-qubit state $\psi$, in terms of expectation values $\langle\vec{S}\rangle_{\psi}$ of collective spin operators $\vec{S}$. For pairwise entanglement, the concurrence $C$ (an entanglement measure introduced by Wootters [12]) was calculated for spin coherent states (SCSs) [13,14], Dicke and Kitagawa-Ueda [15] spin squeezed states, together with mixed states of Heisenberg models. Kitagawa-Ueda [15] spin squeezed states are the spin version of traditional parity-adapted CSs, sometimes called "Schrödinger cat states" since they are a quantum superposition of weakly overlapping (macroscopically distinguishable) quasi-classical coherent wave packets. They were first introduced by Dodonov, Malkin and Man'ko [16] and later adapted to more general finite groups than the parity group $\mathbb{Z}_{2}=\{1,-1\}$ [17]. In this article, we shall introduce $\mathrm{U}(D)$ SCSs (denoted DSCSs for brevity) adapted to the parity symmetry $\mathbb{Z}_{2} \times \stackrel{D-1}{.}$ $\times \mathbb{Z}_{2}$, which are a $D$-dimensional generalization of $U(2)$ Schrödinger cats, and we shall refer to them as DCATs for short. In general, parity-adapted CSs are a special set of "nonclassical" states with interesting statistical properties (see [18-20] for several seminal papers). Parity-adapted DSCSs arise as variational states reproducing the energy and structure of ground states in Lipkin-Meshkov-Glick (LMG) D-level atom models (see [21] and later in Sect. 5).

In Ref. [22], the concurrence $C$ was related to the spin squeezing parameter $\xi^{2}=$ $4\left(\Delta S_{\vec{n}_{\perp}}\right)^{2} / N$ introduced by [15], which measures spin fluctuations in an orthogonal 
direction to the mean value $\vec{n} \propto\langle\vec{S}\rangle$ with minimal variance. Spin squeezing means that $\xi^{2}<1$, that is, when the variance $\left(\Delta S_{\vec{n}_{\perp}}\right)^{2}$ is smaller than the standard quantum limit $S / 2=N / 4$ (with $S$ the spin) attained by (quasi-classical) SCSs. This study shows that spin squeezing is related to pairwise correlation for even and odd parity multiqubit states. Squeezing is in general a redistribution of quantum fluctuations between two noncommuting observables $A$ and $B$ while preserving the minimum uncertainty product $\Delta_{\psi} A \Delta_{\psi} B \geq \frac{1}{2}\left|\langle[A, B]\rangle_{\psi}\right|$. Roughly speaking, it means to partly cancel out fluctuations in one direction at the expense of those enhanced in the "conjugated" direction. For the standard radiation field, it implies the variance relation $(\Delta q)^{2}<1 / 4$ for quadrature (position $q$ and momentum $p$ ) operators. For general $\mathrm{U}(D)$ spin systems of identical $D$-level atoms or "quDits," the situation is more complicated and we shall extend the $D=2$ definition of spin squeezing to general $D$.

Spin squeezing can be created in atom systems by making them to interact with each other for a relatively short time in Kerr-like medium with "twisting" nonlinear Hamiltonians like $H=\lambda S_{x}^{2}$ [15], generating entanglement between them [23,24]. This effective Hamiltonian can be realized in ion traps [25] and has produced fourparticle entangled states [26]. There are also some proposals for two-component BECs [23]. Likewise, the ground state at zero temperature of Hamiltonian critical many-body systems possessing discrete (parity) symmetries also exhibits a cat-like structure. The parity symmetry is spontaneously broken in the thermodynamic limit $N \rightarrow \infty$ and degenerated ground states arise. Parity-adapted coherent states are then good variational states, reproducing the energy of the ground state of these quantum critical models in the thermodynamic limit $N \rightarrow \infty$, namely in matter-field interactions (Dicke model) of two-level [27,28] and three-level [29,30] atoms, BEC [31], U(3) vibron models of molecules [32,33], bilayer quantum Hall systems [34] and (LMG) models for two-level atoms [35-37]. Quantum information (fidelity, entropy, fluctuation, entanglement, etc.) measures have proved to be useful in the analysis of the highly correlated ground state structure of these many-body systems and the identification of critical points across the phase diagram. Special attention must be paid to the deep connection between entanglement, squeezing and quantum phase transitions (QPTs); see $[1,4]$ and references therein.

In this article, we want to explore squeezing and interparticle and interlevel quantum correlations in symmetric multi-quDit systems like the ones described by critical LMG models of identical $D$-level atoms (see, e.g., [21,38-40,40-42] for $D=3$ level atom models). The literature mainly concentrates on two-level atoms, displaying a $\mathrm{U}(2)$ symmetry, which is justified when we make atoms to interact with an external monochromatic electromagnetic field. However, the possibility of polychromatic radiation requires the activation of more atom levels and increases the complexity and richness of the system (see, e.g., [29]). In any case, this also applies to general interacting boson models [43] and multi-mode BECs with two or more boson species. Collective operators generate a $\mathrm{U}(D)$ spin symmetry for the case of $D$-level identical atoms or $D$ boson species (quDits). Recently [21], we have calculated the phase diagram of a three-level LMG atom model. Here, we want to explore the connection between entanglement and squeezing with QPTs for this symmetric multi-qutrit system. For this purpose, we extend to $D$ levels the usual definition of Dicke, parityadapted SCSs and NOON states, and propose linear and von Neumann entropies of 
certain reduced density matrices as a measure of interlevel and interparticle entanglement. We also introduce a generalization of SU(2) spin squeezing to $\mathrm{SU}(D)$.

The organization of the paper is as follows. In Sect. 2, we introduce collective $\mathrm{U}(D)$ spin operators $S_{i j}$, their boson realization, their matrix elements in Fock subspaces of $N$ symmetric quDits, DSCSs and their adaptation to parity ("DCATs"), and a generalization of NOON states to $D$-level systems ("NODONs"). In Sect. 3, we give a brief overview on the concepts and measures of interlevel and interparticle entanglement, considering different bipartitions of the whole system, that we put in practice later in Sect. 6. As entanglement measures, we concentrate on linear (impurity) and von Neumann entropies. We compute entanglement between levels and atoms for DSCSs, DCAT and NODON states. In Sect. 4, we extend Kitagawa-Ueda's definition of SU(2) spin squeezing to $\mathrm{SU}(D)$ and we also connect it with two-quDit entanglement introduced in the previous section. In Sect. 5, we introduce $D$-level Lipkin-Meshkov-Glick (LMG) atom models (we particularize to $D=3$ for simplicity) and study their phase diagram and critical points. In Sect. 6, we analyze the ground state structure of the three-level LMG atom model across the phase diagram with the quantum information measures of Sect. 3 and the SU(3)-spin squeezing parameters of Sect. 4, thus revealing the role of entanglement and squeezing as signatures of quantum phase transitions and detectors of critical points. We only compute linear entropy in Sect. 6, since it is easier to compute than von Neumann entropy and eventually provides similar qualitative information for our purposes; the interested reader can consult Refs. [44,45] for a more general study on the relation between both entropies. Finally, Sect. 7 is devoted to conclusions.

\section{State space, symmetries and collective operator matrix elements}

Those readers acquainted with the boson realization of $\mathrm{U}(D)$ spin operators and coherent states can skim read all the way to equation (16), just to introduce essential notation and necessary formulas. We consider a system of $N$ identical atoms of $D$ levels $(N$ quDits in the quantum information jargon). Let us denote by $E_{i j}=|i\rangle\langle j|$ the Hubbard operator describing a transition from the single-atom level $|j\rangle$ to the level $|i\rangle$, with $i, j=1, \ldots, D$. These are a generalization of Pauli matrices for qubits $(D=2)$, namely $E_{12}=\sigma_{+}, E_{21}=\sigma_{-}, E_{11}-E_{22}=\sigma_{3}$ and $E_{11}+E_{22}=\sigma_{0}$ (the $2 \times 2$ identity matrix). The expectation values of $E_{i j}$ account for complex polarizations or coherences between levels for $i \neq j$ and occupation probability of the level $i$ for $i=j$. The $E_{i j}$ represent the $D^{2}$ step operators of $\mathrm{U}(D)$, whose (Cartan-Weyl) matrices $\left\langle l\left|E_{i j}\right| k\right\rangle=\delta_{i l} \delta_{j k}$ fulfill the commutation relations

$$
\left[E_{i j}, E_{k l}\right]=\delta_{j k} E_{i l}-\delta_{i l} E_{k j}
$$

Let us denote by $E_{i j}^{\mu}, \mu=1, \ldots, N$ the embedding of the single $\mu$-th atom $E_{i j}$ operator into the $N$-atom Hilbert space; namely, $E_{i j}^{3}=\mathbb{1}_{D} \otimes \mathbb{1}_{D} \otimes E_{i j} \otimes \mathbb{1}_{D}$ for $N=4$, with $\mathbb{1}_{D}$ the $D \times D$ identity matrix. The collective $\mathrm{U}(D)$-spin $(D$-spin for short) operators are 


$$
S_{i j}=\sum_{\mu=1}^{N} E_{i j}^{\mu}, \quad i, j=1, \ldots, D .
$$

They are the generators of the underlying $U(D)$ dynamical symmetry with the same commutation relations as those of $E_{i j}$ in (1). When focusing on two levels $i>j$, we might prefer to use

$$
\begin{aligned}
& \vec{J}^{(i j)}=\left(J_{x}^{(i j)}, J_{y}^{(i j)}, J_{z}^{(i j)}\right), \quad J_{x}^{(i j)}=\frac{S_{i j}+S_{j i}}{2}, \\
& J_{y}^{(i j)}=\mathrm{i} \frac{S_{i j}-S_{j i}}{2}, \quad J_{z}^{(i j)}=\frac{S_{j j}-S_{i i}}{2},
\end{aligned}
$$

(roman i denotes the imaginary unit throughout the article) with commutation relations $\left[J_{x}^{(i j)}, J_{y}^{(i j)}\right]=\mathrm{i} J_{z}^{(i j)}$ (and cyclic permutations of $x, y, z$ ), which is an embedding of $D(D-1) / 2 \mathrm{SU}(2)$ subalgebras into $\mathrm{U}(D)$. Although the form (3) for $D$-spin operators could be more convenient to extrapolate all the $D=2$ level machinery to arbitrary $D$, we shall still prefer the form (2) (at least in this paper), which allows for more compact formulas.

The $D^{N}$-dimensional Hilbert space is the $N$-fold tensor product $\left[\mathbb{C}^{D}\right]^{\otimes N}$. The tensor product representation of $\mathrm{U}(D)$ is reducible and decomposes into a ClebschGordan direct sum of mixed symmetry invariant subspaces. Here, we shall restrict ourselves to the $\left(\begin{array}{c}N+D-1 \\ N\end{array}\right)$-dimensional fully symmetric sector (see [21] for the role of other mixed symmetry sectors), which means that our $N$ atoms are indistinguishable (bosons). Denoting by $a_{i}^{\dagger}$ (resp. $a_{i}$ ) the creation (resp. annihilation) operator of an atom in the $i$-th level, the collective $D$-spin operators (2) can be expressed (in this fully symmetric case) as bilinear products of creation and annihilation operators as (Schwinger representation)

$$
S_{i j}=a_{i}^{\dagger} a_{j}, \quad i, j=1, \ldots, D
$$

$S_{i i}$ is the operator number of atoms at level $i$, whereas $S_{i j}, i \neq j$ are raising and lowering operators. The fully symmetric representation space of $\mathrm{U}(D)$ is embedded into Fock space, with Bose-Einstein-Fock basis $(|\overrightarrow{0}\rangle$ denotes the Fock vacuum)

$$
|\vec{n}\rangle=\left|n_{1}, \ldots, n_{D}\right\rangle=\frac{\left(a_{1}^{\dagger}\right)^{n_{1}} \ldots\left(a_{D}^{\dagger}\right)^{n_{D}}}{\sqrt{n_{1} ! \ldots n_{D} !}}|\overrightarrow{0}\rangle
$$

when fixing $n_{1}+\cdots+n_{D}=N$ (the linear Casimir $C_{1}=S_{11}+\cdots+S_{D D}$ ) to the total number $N$ of atoms. There are other realizations of $D$-spin operators in terms of more than $D$ bosonic modes (e.g., when each level $j$ contains $M$ degenerate orbitals), which describe mixed symmetries [46-48], but we shall not consider them here. 
Collective $D$-spin operators (4) matrix elements are given by

$$
\begin{aligned}
& \left\langle\vec{m}\left|S_{i j}\right| \vec{n}\right\rangle=\sqrt{\left(n_{i}+1\right) n_{j}} \delta_{m_{i}, n_{i}+1} \delta_{m_{j}, n_{j}-1} \prod_{k \neq i, j}^{D} \delta_{m_{k}, n_{k}}, \forall i \neq j, \\
& \left\langle\vec{m}\left|S_{i i}\right| \vec{n}\right\rangle=n_{i} \delta_{\vec{m}, \vec{n}} .
\end{aligned}
$$

The expansion of a general symmetric $N$-particle state $\psi$ in the Fock basis will be written as

$$
|\psi\rangle=\sum_{\vec{n}}^{\prime} c_{\vec{n}}|\vec{n}\rangle=\sum_{n_{1}+\cdots+n_{D}=N} c_{n_{1}, \ldots, n_{D}}\left|n_{1}, \ldots, n_{D}\right\rangle
$$

where $\sum^{\prime}$ is a shorthand for the restricted sum. $D$-spin operator expectation values (EVs) can then be easily computed as

$$
\left\langle S_{i j}\right\rangle_{\psi}=\left\langle\psi\left|S_{i j}\right| \psi\right\rangle=\sum_{\vec{n}}{ }^{\prime} \bar{c}_{\vec{n}_{i j}} c_{\vec{n}} \sqrt{\left(n_{i}+1\right) n_{j}}, i \neq j, \quad\left\langle S_{i i}\right\rangle_{\psi}=\sum_{\vec{n}}{ }^{\prime} n_{i}\left|c_{\vec{n}}\right|^{2},
$$

where we have used (6) and where by $\vec{n}_{i j}$ we mean to replace $n_{i} \rightarrow n_{i}+1$ and $n_{j} \rightarrow n_{j}-1$ in $\vec{n}$.

Among all symmetric multi-quDit states, we shall pay special attention to $\mathrm{U}(D)$ SCSs (DSCSs for short)

$$
|\mathbf{z}\rangle=\left|\left(z_{1}, z_{2}, \ldots, z_{D}\right)\right\rangle=\frac{1}{\sqrt{N !}}\left(\frac{z_{1} a_{1}^{\dagger}+z_{2} a_{2}^{\dagger}+\cdots+z_{D} a_{D}^{\dagger}}{\sqrt{\left|z_{1}\right|^{2}+\left|z_{2}\right|^{2}+\cdots+\left|z_{D}\right|^{2}}}\right)^{N}|\overrightarrow{0}\rangle
$$

which are labeled by complex points $\mathbf{z}=\left(z_{1}, \ldots, z_{D}\right) \in \mathbb{C}^{D}$. To be more precise, there is an equivalence relation: $\left|\mathbf{z}^{\prime}\right\rangle \sim|\mathbf{z}\rangle$ if $\mathbf{z}^{\prime}=q \mathbf{z}$ for any complex number $q \neq 0$, which means that $|\mathbf{z}\rangle$ is actually labeled by class representatives of complex lines in $\mathbb{C}^{D}$, that is, by points of the complex projective phase space

$$
\mathbb{C} P^{D-1}=\left[\mathbb{C}^{D} / \sim\right]=\mathrm{U}(D) /[\mathrm{U}(1) \times \mathrm{U}(D-1)] .
$$

A class/coset representative can be chosen as $\tilde{\mathbf{z}}=\mathbf{z} / z_{i}$ when $z_{i} \neq 0$, which corresponds to a certain patch of the manifold $\mathbb{C} P^{D-1}$. This is equivalent to choose $i$ as a reference level and set $z_{i}=1$. For the moment, we shall allow redundancy in $\mathbf{z}$ to write general expressions, although we shall eventually take $i=1$ as a reference (lower energy) level and set $z_{1}=1$ in Sect. 5 .

DSCSs (multi-nomial) can be seen as BECs of $D$ modes, generalizing the spin U(2) (binomial) coherent states of two modes introduced by [13] and [14] long ago. For $\mathbf{z}=\mathbf{e}_{i}$ (the standard/canonical basis vectors of $\mathbb{C}^{D}$ ), the DSCS $\left|\mathbf{e}_{i}\right\rangle=\left(a_{i}^{\dagger}\right)^{N}|\overrightarrow{0}\rangle / \sqrt{N !}$ 
corresponds to a BEC of $N$ atoms placed at level $i{ }^{1}$ If we order levels $i=1, \ldots, D$ from lower to higher energies, the state $\left|\mathbf{e}_{1}\right\rangle$ would be the ground state, whereas general $|\mathbf{z}\rangle$ could be seen as coherent excitations. Coherent states are sometimes called "quasiclassical" states and we shall see in Sect. 5 that $|\mathbf{z}\rangle$ turns out to be a good variational state that reproduces the energy and wave function of the ground state of multi-level LMG atom models in the thermodynamic (classical) limit $N \rightarrow \infty$.

Expanding the multi-nomial (9), we identify the coefficients $c_{\vec{n}}$ of the expansion (7) of the DSCS $|\mathbf{z}\rangle$ in the Fock basis as

$$
c_{\vec{n}}(\mathbf{z})=\sqrt{\frac{N !}{\prod_{i=1}^{D} n_{i} !}} \frac{\prod_{i=1}^{D} z_{i}^{n_{i}}}{|\mathbf{z}|^{N}}
$$

where we have written $|\mathbf{z}|=(\mathbf{z} \cdot \mathbf{z})^{1 / 2}=\left(\sum_{i=1}^{D}\left|z_{i}\right|^{2}\right)^{1 / 2}$ for the length of $\mathbf{z}$. Note that DSCS are not orthogonal (in general) since

$$
\left\langle\mathbf{z}^{\prime} \mid \mathbf{z}\right\rangle=\frac{\left(\mathbf{z}^{\prime} \cdot \mathbf{z}\right)^{N}}{\left(\mathbf{z}^{\prime} \cdot \mathbf{z}^{\prime}\right)^{N / 2}(\mathbf{z} \cdot \mathbf{z})^{N / 2}}, \quad \mathbf{z}^{\prime} \cdot \mathbf{z}=\bar{z}_{1}^{\prime} z_{1}+\cdots+\bar{z}_{D}^{\prime} z_{D}
$$

However, contrary to the standard CSs, they can be orthogonal when $\mathbf{z}^{\prime} \cdot \mathbf{z}=0$. EVs of $D$-spin operators $S_{i j}$ (coherences for $i \neq j$ and mean level populations for $i=j$ ) in a DSCS are simply written as

$$
\left\langle S_{i j}\right\rangle_{\mathbf{z}}=\left\langle\mathbf{z}\left|S_{i j}\right| \mathbf{z}\right\rangle=N \bar{z}_{i} z_{j} /|\mathbf{z}|^{2}
$$

DSCS non-diagonal matrix elements of $D$-spin operators can also be compactly written as

$$
\left\langle\mathbf{z}^{\prime}\left|S_{i j}\right| \mathbf{z}\right\rangle=N \bar{z}_{i}^{\prime} z_{j} \frac{\left(\mathbf{z}^{\prime} \cdot \mathbf{z}\right)^{N-1}}{\left|\mathbf{z}^{\prime}\right|^{N}|\mathbf{z}|^{N}}
$$

Similarly, EVs of quadratic powers of $D$-spin operators in a DSCS state can be concisely written as

$$
\left\langle\mathbf{z}\left|S_{i j} S_{k l}\right| \mathbf{z}\right\rangle=\frac{\bar{z}_{i} z_{l}}{|\mathbf{z}|^{4}}\left(N \delta_{j k}|\mathbf{z}|^{2}+N(N-1) \bar{z}_{k} z_{j}\right),
$$

and their DSCS matrix elements as

$$
\left\langle\mathbf{z}^{\prime}\left|S_{i j} S_{k l}\right| \mathbf{z}\right\rangle=\frac{\bar{z}_{i}^{\prime} z_{l}}{\left|\mathbf{z}^{\prime}\right|^{N}|\mathbf{z}|^{N}}\left(N \delta_{j k}\left(\mathbf{z}^{\prime} \cdot \mathbf{z}\right)^{N-1}+N(N-1) \bar{z}_{k}^{\prime} z_{j}\left(\mathbf{z}^{\prime} \cdot \mathbf{z}\right)^{N-2}\right) .
$$

\footnotetext{
${ }^{1}$ Note the difference between Fock states $\left|n_{1}, \ldots, n_{D}\right\rangle$ and DSCSs $\left|\left(z_{1}, \ldots, z_{D}\right)\right\rangle$, which are placed inside parentheses to avoid confusion. For instance, $\left|\mathbf{e}_{i}\right\rangle=|(0, \ldots, 1, \ldots, 0)\rangle=\left(a_{i}^{\dagger}\right)^{N}|\overrightarrow{0}\rangle / \sqrt{N !}=$ $|0, \ldots, N, \ldots, 0\rangle$.
} 
Note that, for large $N$, quantum fluctuations are negligible and we have $\left\langle\mathbf{z}\left|S_{i j} S_{k l}\right| \mathbf{z}\right\rangle \simeq$ $\left\langle\mathbf{z}\left|S_{i j}\right| \mathbf{z}\right\rangle\left\langle\mathbf{z}\left|S_{k l}\right| \mathbf{z}\right\rangle$. Otherwise stated, in the thermodynamical (classical) limit we have

$$
\lim _{N \rightarrow \infty} \frac{\left\langle\mathbf{z}\left|S_{i j} S_{k l}\right| \mathbf{z}\right\rangle}{\left\langle\mathbf{z}\left|S_{i j}\right| \mathbf{z}\right\rangle\left\langle\mathbf{z}\left|S_{k l}\right| \mathbf{z}\right\rangle}=1 .
$$

We shall use these ingredients when computing one- and two-quDit RDMs in the next section.

We shall see that DSCSs are separable and exhibit no atom entanglement (although they do exhibit level entanglement). The situation changes when we deal with parity-adapted DSCSs, sometimes called "Schrödinger cat states" (commented at the introduction), since they are a quantum superposition of weakly overlapping (macroscopically distinguishable) quasi-classical coherent wave packets, as we shall explicitly see below. These kind of cat states arise in several interesting physical situations. As we have already mentioned, they can be generated via amplitude dispersion by evolving CSs in Kerr media, with a strong nonlinear interaction, like the already commented spin-squeezed states of [15]. They exhibit statistical properties similar to squeezed states, with deviations from Poissonian (CS) distributions. Squeezing and multi-particle entanglement are important quantum resources that make Schrödinger cats useful for quantum enhanced metrology [2]. They are also good variational states [14], reproducing the energy of the ground state of quantum critical models in the thermodynamic limit $N \rightarrow \infty$. To construct them, we require parity operators defined as

$$
\Pi_{j}=\exp \left(\mathrm{i} \pi S_{j j}\right), \quad j=1, \ldots, D .
$$

They are conserved when the Hamiltonian scatters pairs of particles conserving the parity of the population $n_{j}$ in each level $j=1, \ldots, D$. It is easy to see that $\Pi_{j}\left(a_{j}^{\dagger}\right)^{n_{j}}|\overrightarrow{0}\rangle=\left(-a_{j}^{\dagger}\right)^{n_{j}}|\overrightarrow{0}\rangle$, so that the effect of parity operations on number states (5) is $\Pi_{j}|\vec{n}\rangle=(-1)^{n_{j}}|\vec{n}\rangle$. Likewise, using the multi-nomial expansion (9), it is easy to see that the effect of parity operators on symmetric DSCSs $|\mathbf{z}\rangle$ is then

$$
\Pi_{i}|\mathbf{z}\rangle=\Pi_{i}\left|\left(z_{1}, \ldots, z_{i}, \ldots, z_{D}\right)\right\rangle=\left|\left(z_{1}, \ldots,-z_{i}, \ldots, z_{D}\right)\right\rangle .
$$

Note that $\Pi_{i}^{-1}=\Pi_{i}$ and $\Pi_{1} \ldots \Pi_{D}=(-1)^{N}$, a constraint that says that the parity group for symmetric quDits is not $\mathbb{Z}_{2} \times . . . \times \mathbb{Z}_{2}$ but $\mathbb{Z}_{2} \times \stackrel{D-1}{\cdots} \times \mathbb{Z}_{2}$ instead. In order to define a projector on definite parity (even or odd), we have to choose a reference level, namely $i=1$. Doing so, the projector on even parity becomes

$$
\Pi_{\text {even }}=2^{1-D} \sum_{\mathfrak{b} \in\{0,1\}^{D-1}} \Pi_{2}^{b_{2}} \Pi_{3}^{b_{3}} \cdots \Pi_{D}^{b_{D}},
$$

where we denote the binary string $\mathfrak{b}=\left(b_{2}, \ldots, b_{D}\right) \in\{0,1\}^{D-1}$. Likewise, the projection operator on odd parity is $\Pi_{\text {odd }}=\mathbb{1}-\Pi_{\text {even }}$. Choosing level $i=1$ as a reference level is equivalent to choose a patch on the manifold $\mathbb{C} P^{D-1}$ where $z_{1} \neq 0$; 
in this way, any coherent state $|\mathbf{z}\rangle$ is equivalent to the class representative $\left|\mathbf{z} / z_{1}\right\rangle$, due to equivalence relation $\left|\mathbf{z}^{\prime}\right\rangle \sim|\mathbf{z}\rangle$ if $\mathbf{z}^{\prime}=q \mathbf{z}$ with $q \neq 0$. Let us simply denote by $\mathbf{z}=\left(1, z_{2}, \ldots, z_{D}\right)$ the class representative in this case. It will be useful, for later use as variational ground states, to define the (unnormalized) generalized Schrödinger even cat state

$$
\mid \text { DCAT }\}=\Pi_{\text {even }}|\mathbf{z}\rangle=2^{1-D} \sum_{\mathfrak{b}}\left|\mathbf{z}^{\natural}\right\rangle
$$

where $\mathbf{z}^{\mathfrak{b}}=\left(1,(-1)^{b_{2}} z_{2}, \ldots,(-1)^{b_{D}} z_{D}\right)$ and we are using $\sum_{\mathfrak{b}}$ as a shorthand for $\sum_{b \in\{0,1\}^{D-1}}$. It is just the projection of a DSCS on the even parity subspace. The state (20) is a generalization of the even cat state for $D=2$ in the literature [16], given by

$$
\mid 2 \mathrm{CAT}\}=\frac{1}{2}(|(1, \alpha)\rangle+|(1,-\alpha)\rangle)
$$

for the class representative $\mathbf{z}=\left(z_{1}, z_{2}\right)=(1, \alpha)$. The shorthand $|\alpha\rangle=|(1, \alpha)\rangle$ is used in the literature when a class representative (related to highest $\left|\mathbf{e}_{1}\right\rangle$ or lowest $\left|\mathbf{e}_{2}\right\rangle$ weight fiducial vectors) is implicitly chosen. The squared norm of $\mid 2 \mathrm{CAT}\}$ is simply

$$
\mathcal{N}(2 \mathrm{CAT})^{2}=\{2 \mathrm{CAT} \mid 2 \mathrm{CAT}\}=\frac{1}{2}\left[1+\left(\frac{1-|\alpha|^{2}}{1+|\alpha|^{2}}\right)^{N}\right] .
$$

Note that the overlap $\langle(1, \alpha) \mid(1,-\alpha)\rangle=\left(\left(1-|\alpha|^{2}\right) /\left(1+|\alpha|^{2}\right)\right)^{N} \stackrel{N \rightarrow \infty}{\longrightarrow} 0$, which means that $|(1, \alpha)\rangle$ and $|(1,-\alpha)\rangle$ are macroscopically distinguishable wave packets for any $\alpha$ (they are orthogonal for $|\alpha|=1$ ). Likewise, the unnormalized 3CAT is explicitly given by

$$
\mid 3 \mathrm{CAT}\}=\frac{1}{4}(|(1, \alpha, \beta)\rangle+|(1,-\alpha, \beta)\rangle+|(1, \alpha,-\beta)\rangle+|(1,-\alpha,-\beta)\rangle)
$$

when setting $\mathbf{z}=\left(z_{1}, z_{2}, z_{3}\right)=(1, \alpha, \beta)$ as a class representative. The squared norm is now

$$
\mathcal{N}(3 \mathrm{CAT})^{2}=\frac{1}{4}\left[1+\frac{\left(1-|\alpha|^{2}+|\beta|^{2}\right)^{N}+\left(1+|\alpha|^{2}-|\beta|^{2}\right)^{N}+\left(1-|\alpha|^{2}-|\beta|^{2}\right)^{N}}{\left(1+|\alpha|^{2}+|\beta|^{2}\right)^{N}}\right] .
$$

These expressions can be generalized to arbitrary $D$ as

$$
\mathcal{N}(\text { DCAT })^{2}=2^{1-D} \frac{\sum_{\mathfrak{b}}\left(\mathbf{z}^{\mathfrak{b}} \cdot \mathbf{z}\right)^{N}}{|\mathbf{z}|^{2 N}} .
$$

We shall use (23) and (24) in Sects. 5 and 6, when discussing a LMG model of atoms with $D=3$ levels. These 3CAT states have also been used in U(3) vibron 
models of molecules [32,33] and Dicke models of three-level atoms interacting with a polychromatic radiation field [29,30].

The $D$-spin EVs on a DCAT state (20) can be now computed and the general expression is

$$
\left\langle\text { DCAT }\left|S_{i j}\right| \mathrm{DCAT}\right\rangle=N \delta_{i j} \frac{\sum_{\mathfrak{b}}(-1)^{b_{i}}\left|z_{i}\right|^{2}\left(\mathbf{z}^{\mathfrak{b}} \cdot \mathbf{z}\right)^{N-1}}{\sum_{\mathfrak{b}}\left(\mathbf{z}^{\mathfrak{b}} \cdot \mathbf{z}\right)^{N}},
$$

where we set $(-1)^{b_{i}}=1=\left|z_{i}\right|$ for $i=1$ (reference level). Similarly, EVs of quadratic powers of $D$-spin operators in a DCAT state can be concisely written as

$$
\begin{aligned}
\left\langle\mathrm{DCAT}\left|S_{i j} S_{k l}\right| \mathrm{DCAT}\right\rangle= & N\left(\delta_{i j} \delta_{k l}+\delta_{i k} \delta_{j l}+\delta_{i l} \delta_{j k}-2 \delta_{i j} \delta_{j k} \delta_{k l} \delta_{l i}\right) \\
& \times \frac{\sum_{\mathfrak{b}}(-1)^{b_{i}} \bar{z}_{i} z_{l}\left[\delta_{j k}\left(\mathbf{z}^{\mathrm{b}} \cdot \mathbf{z}\right)^{N-1}+(N-1)(-1)^{b_{k}} \bar{z}_{k} z_{j}\left(\mathbf{z}^{\mathrm{b}} \cdot \mathbf{z}\right)^{N-2}\right]}{\sum_{\mathfrak{b}}\left(\mathbf{z}^{\mathrm{b}} \cdot \mathbf{z}\right)^{N}} .
\end{aligned}
$$

To finish this section, let us comment on a generalization to arbitrary $D$ of another prominent example of quantum states that are useful for quantum-enhanced metrology and provide phase sensitivities beyond the standard quantum limit. We refer to Greenberger-Horne-Zeilinger (GHZ) or "NOON" (when considering bosonic particles) states. For $D=2$ level systems, NOON states can be written in the Fock state notation (5) as (see, e.g., [2])

$$
\mid \text { NOON }\rangle=\frac{1}{\sqrt{2}}\left(|N, 0\rangle+e^{\mathrm{i} \phi}|0, N\rangle\right)=\frac{1}{\sqrt{2}}\left(\frac{\left(a_{1}^{\dagger}\right)^{N}}{\sqrt{N !}}|\overrightarrow{0}\rangle+e^{\mathrm{i} \phi} \frac{\left(a_{2}^{\dagger}\right)^{N}}{\sqrt{N !}}|\overrightarrow{0}\rangle\right) .
$$

Using the canonical basis vectors $\left\{\mathbf{e}_{1}, \mathbf{e}_{2}\right\}$ of $\mathbb{C}^{2}$, we can write the NOON state as a linear superposition of U(2) SCSs

$$
|\mathrm{NOON}\rangle=\frac{1}{\sqrt{2}}\left(e^{\mathrm{i} \phi_{1}}\left|\mathbf{e}_{1}\right\rangle+e^{\mathrm{i} \phi_{2}}\left|\mathbf{e}_{2}\right\rangle\right),
$$

with phases $e^{\mathrm{i} \phi_{1,2}}$, which coincides with (28) (up to an irrelevant global phase $e^{\mathrm{i} \phi_{1}}$ ) for the relative phase $\phi=\phi_{2}-\phi_{1}$. Multi-mode (or multi-level, in our context) generalizations of NOON states have been proposed in the literature (see, e.g., [49,50]). In our scheme, this generalization of NOON states (29) to $D$-level systems adopts the following form

$$
\begin{aligned}
\mid \text { NODON }\rangle= & \frac{1}{\sqrt{D}}\left(\sum_{j=1}^{D} e^{\mathrm{i} \phi_{j}}\left|\mathbf{e}_{j}\right\rangle\right) \\
= & \frac{1}{\sqrt{D}}\left(e^{\mathrm{i} \phi_{1}}|N, 0, \ldots\rangle+e^{\mathrm{i} \phi_{2}}|0, N, 0, \ldots\rangle\right. \\
& \left.+\cdots+e^{\mathrm{i} \phi_{D}}|0,0, \ldots, N\rangle\right) .
\end{aligned}
$$


EVs of linear and quadratic powers of $D$-spin operators in NODON states can be easily calculated as

$$
\left\langle\text { NODON }\left|S_{i j}\right| \text { NODON }\right\rangle=\frac{N}{D} \delta_{i j}, \quad\left\langle\text { NODON }\left|S_{i j} S_{k l}\right| \text { NODON }\right\rangle=\frac{N}{D} \delta_{i l}\left(\delta_{j k}+(N-1) \delta_{i k} \delta_{i j}\right) .
$$

The computations in this section will be necessary to discuss entanglement and squeezing properties of all these states in Sects. 3 and 4.

\section{Entanglement measures in multi-quDit systems}

In this section, we define several types of bipartition of the whole system, computing the corresponding RDMs and entanglement measures for different kinds of symmetric multi-quDit states $\psi$ in terms of linear $\mathscr{L}$ and von Neumann $\mathcal{S}$ entropies. We start computing interlevel entanglement in Sect. 3.1 and then (one- and two-quDit) interparticle entanglement in Sect. 3.2.

\subsection{Entanglement among levels}

For a general symmetric $N$-particle state $\psi$ like (7), the RDM on the level $i$ is

$$
\varrho_{i}(\psi)=\operatorname{tr}_{j \neq i}\left(\sum_{\vec{n}, \vec{n}^{\prime}}{ }^{\prime} c_{\vec{n}^{\prime}} \bar{c}_{\vec{n}}\left|n_{1}^{\prime}, \ldots, n_{D}^{\prime}\right\rangle\left\langle n_{1}, \ldots, n_{D}\right|\right)=\sum_{\vec{n}}{ }^{\prime}\left|c_{\vec{n}}\right|^{2}\left|n_{i}\right\rangle\left\langle n_{i}\right|
$$

Thus, $\varrho_{i}(\psi)$ lies in a single boson Hilbert space of dimension $N+1$. Its purity is then

$$
\mathscr{P}_{i}^{\ell}(\psi)=\operatorname{tr}\left(\varrho_{i}^{2}(\psi)\right)=\sum_{\vec{n}, \vec{m}}\left|c_{\vec{n}}\right|^{2}\left|c_{\vec{m}}\right|^{2} \delta_{n_{i}, m_{i}}
$$

Here, the superscript $\ell$ makes reference to "level," to distinguish it from "atom" purity $\mathscr{P}^{\mathrm{a}}$ in the next section. It can also make reference to entanglement between different boson species $\ell$, like rotational-vibrational entanglement [32,33] in algebraic molecular models [51,52] such as the vibron model based on a bosonic U(3) spectrum-generating algebra [53,54]. For the case of the DSCS $|\mathbf{z}\rangle$ in (9), taking the coefficients $c_{\vec{n}}$ in (10), and after a lengthy calculation, the RDM on level $i$ turns out to be diagonal

$$
\begin{aligned}
\varrho_{i}(\mathbf{z}) & =\sum_{n=0}^{N} \lambda_{n}\left(x_{i}, y_{i}\right)|N-n\rangle\langle N-n|, \\
\lambda_{n}\left(x_{i}, y_{i}\right) & =\left(\begin{array}{c}
N \\
n
\end{array}\right) \frac{x_{i}^{N-n} y_{i}^{n}}{\left(x_{i}+y_{i}\right)^{N}}, \quad x_{i}=\left|z_{i}\right|^{2}, y_{i}=|\mathbf{z}|^{2}-\left|z_{i}\right|^{2} .
\end{aligned}
$$



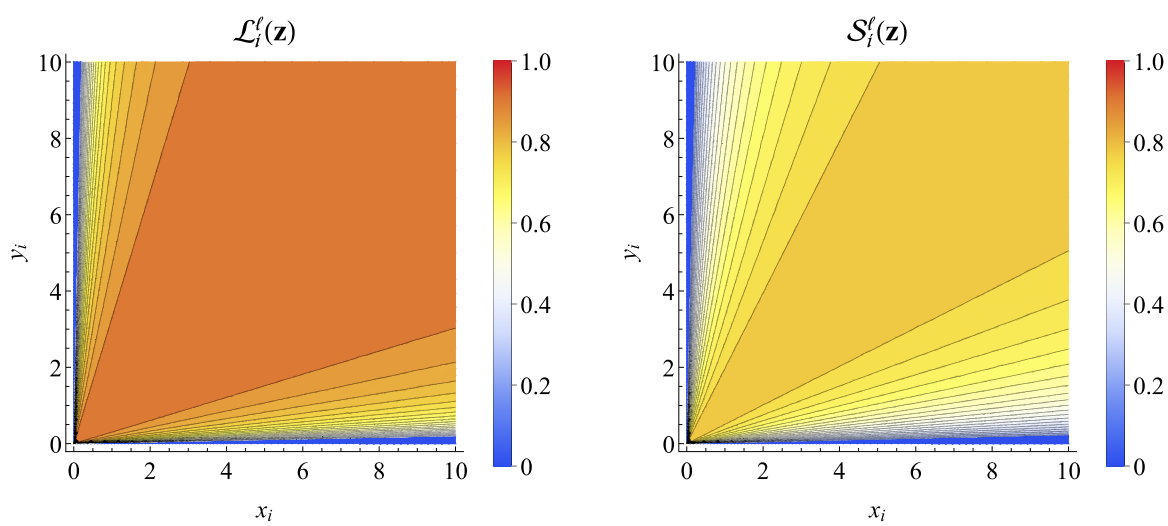

Fig. 1 Contour plots of the linear $\mathscr{L}_{i}^{\ell}$ and von Neumann $\delta_{i}^{\ell}$ entanglement entropies, associated with the $\mathrm{RDM}$ of a $\mathrm{U}(D)$-spin coherent state $|\mathbf{z}\rangle$ of $N=10$ quDits on level $i$ (34), as a function of the phase-space coordinates $(x, y)$

Note that the eigenvalues $\lambda_{n}$ can be expressed in terms of only two positive real coordinates $\left(x_{i}, y_{i}\right)$, except for the reference level $z_{i}=1$, for which $x_{i}=1$ and therefore there is only one independent variable $y_{i}=|\mathbf{z}|^{2}-1$. For example, for $\mathrm{U}(3)$ SCSs, choosing $i=1$ as the reference level and using the parametrization $\mathbf{z}=(1, \alpha, \beta)$ for the phase space $\mathbb{C} P^{2}$ in (23), we have $x_{1}=1, x_{2}=|\alpha|^{2}, x_{3}=|\beta|^{2}$ and $y_{1}=|\alpha|^{2}+|\beta|^{2}, y_{2}=1+|\beta|^{2}, y_{3}=1+|\alpha|^{2}$. The purity of $\varrho_{i}(\mathbf{z})$ is simply $\mathscr{P}_{i}^{\ell}\left(x_{i}, y_{i}\right)=\sum_{n=0}^{N} \lambda_{n}^{2}\left(x_{i}, y_{i}\right)$. In Fig. 1, we represent the linear and von Neumann

$$
\mathscr{L}_{i}^{\ell}=\frac{N+1}{N}\left(1-\mathscr{P}_{i}^{\ell}\right), \quad \delta_{i}^{\ell}=-\sum_{n=0}^{N} \lambda_{n} \log _{N+1} \lambda_{n}
$$

entanglement entropies for a general level $i$ as a function of $(x, y)$ [for the reference level $i=1$, we have to restrict ourselves to the cross section $x=1]$. We normalize linear and von Neumann entropies so that their interval range is $[0,1]$, the extremal values corresponding to pure and completely mixed RDMs, respectively. We shall see that both entropies, $\mathscr{L}$ and $\mathcal{S}$, provide similar qualitative behavior for the bipartitions studied in this paper. Interlevel isentropic contours correspond to the straight lines $y=m x$ (see Fig. 1), and the maximum is attained for $y=x$. The large $N$ behavior of the interlevel linear entanglement entropy $\mathscr{L}_{i}^{\ell}(\mathbf{z})$ around the maximum $y=x$ is $\mathscr{L}_{i}^{\ell}=1-1 / \sqrt{\pi N}+O\left(N^{-3 / 2}\right)$.

In Fig. 2, we represent contour plots of $\mathscr{L}_{1,2}^{\ell}$ and $\delta_{1,2}^{\ell}$ for the RDM of a 3CAT of $N=20$ qutrits on levels $i=1$ and $i=2$. Note that linear and von Neumann entropies display a similar structure. We omit $\mathscr{L}_{3}^{\ell}$ and $\delta_{3}^{\ell}$ since they are just the reflection in a diagonal mirror line of $\mathscr{L}_{2}^{\ell}$ and $\delta_{2}^{\ell}$, respectively. $\mathscr{L}_{1}^{\ell}$ attains its maximum at the isentropic circle $|\alpha|^{2}+|\beta|^{2}=1$, whereas $\mathscr{L}_{2}^{\ell}$ attains its maximum at the isentropic hyperbola $|\alpha|^{2}-|\beta|^{2}=1$. The large $N$ behavior of the interlevel linear entanglement entropy for a DCAT around the maximum is $\mathscr{L}_{i}^{\ell}=1-2 / \sqrt{\pi N}+O\left(N^{-3 / 2}\right)$. Figure 2 also shows (in magenta color) the parametric curve $(\alpha(\lambda), \beta(\lambda))$ obtained later in 

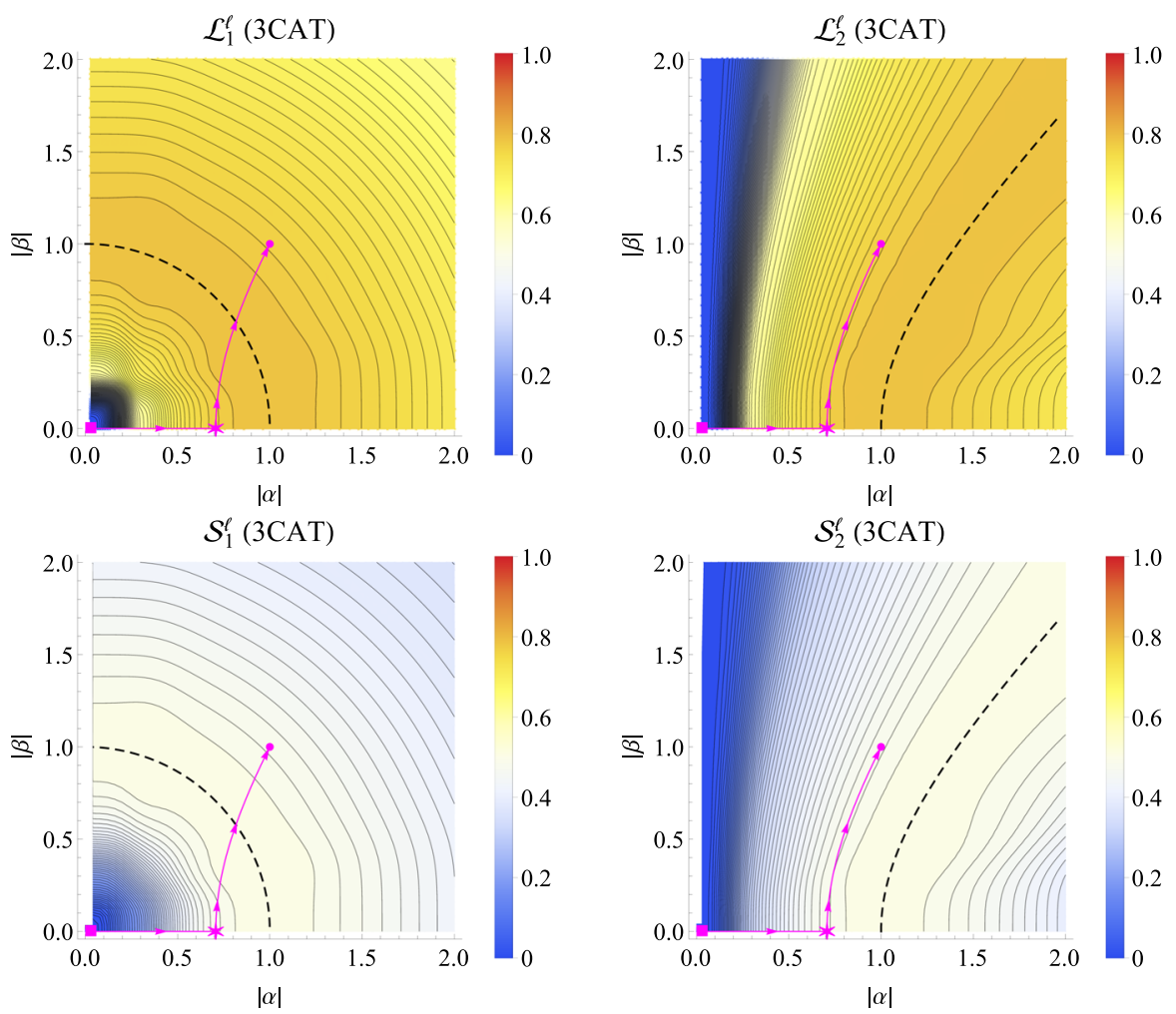

Fig. 2 Contour plots of the linear $\mathscr{L}_{i}^{\ell}$ and von Neumann $\delta_{i}^{\ell}$ entanglement entropies, associated with the RDM of a 3CAT of $N=20$ qutrits on levels $i=1$ and $i=2$ (the case $i=3$ is just the reflection in a diagonal mirror line of $i=2$ ), as a function of the phase-space coordinates $(\alpha, \beta)$ (they just depend on moduli). Dashed contours represent maximum entanglement entropy. The meaning of the magenta curve is explained in the main text

Sect. 5 and related to the stationary points (59) of the energy surface (57) in the quantum phase diagram of a LMG three-level atom model, where $\lambda$ is the atom-atom interaction coupling constant. For high interactions we have $(\alpha(\lambda), \beta(\lambda)) \stackrel{\lambda \rightarrow \infty}{\longrightarrow}(1,1)$, which does not lie inside the maximum isentropic curve, although the difference between both entropies tends to zero in the limit $N \rightarrow \infty$ (see later in Sects. 5 and 6 for more information).

For NODON states (30), the RDM on level $i$ and its purity are

$$
\varrho_{i}(\mathrm{NODON})=\frac{D-1}{D}|0\rangle_{i}\left\langle 0\left|+\frac{1}{D}\right| N\right\rangle_{i}\langle N|, \quad \operatorname{tr}\left(\varrho_{i}^{2}(\mathrm{NODON})\right)=1-2\left(\frac{D-1}{D^{2}}\right),
$$

which is independent of the level $i$. Therefore, the linear entropy is given by $\mathscr{L}_{i}^{\ell}(\mathrm{NODON})=2 \frac{N+1}{N} \frac{D-1}{D^{2}}$, which reduces to $\mathscr{L}_{i}^{\ell}(\mathrm{NOON})=\frac{N+1}{2 N}$ for $D=2$. 


\subsection{Entanglement among atoms}

We compute the one- and two-particle RDMs for a single and a pair of particles extracted at random from a symmetric $N$-quDit state. The corresponding entanglement entropies are expressed in terms of EVs of collective $D$-spin operators $S_{i j}$.

\subsubsection{One-quDit reduced density matrices}

Any density matrix of a single quDit can be written as a combination of Hubbard matrices $E_{i j}$ with commutation relations (1) as

$$
\rho_{1}=\sum_{i, j=1}^{D} r_{i j} E_{i j}, \quad r_{i j}=\operatorname{tr}\left(\rho_{1} E_{j i}\right)=\left\langle E_{j i}\right\rangle
$$

with $r_{i j}$ complex numbers (the generalized Bloch vector) fulfilling $\bar{r}_{i j}=r_{j i}$ and (the generalized Bloch sphere)

$$
\operatorname{tr}\left[\rho_{1}\right]=\sum_{i=1}^{D} r_{i i}=1, \quad 0<\operatorname{tr}\left[\left(\rho_{1}\right)^{2}\right]=\sum_{i, j=1}^{D}\left|r_{i j}\right|^{2} \leq 1
$$

We want to construct the one-quDit RDM for one quDit extracted at random from a symmetric $N$-quDit state $\psi$. The procedure consists of writing the one-quDit RDM entries in terms of expectation values (EVs) of collective $D$-spin operators (2). Remember the definition of $E_{i j}^{\mu}, \mu=1, \ldots, N$ after (1) as the embedding of the single $\mu$-th atom $E_{i j}$ operator into the $N$-atom Hilbert space. Atom indistinguishableness implies that $\left\langle E_{i j}^{\mu}\right\rangle=\frac{1}{N}\left\langle S_{i j}\right\rangle$, for any $\mu=1, \ldots, N$, and therefore the one-quDit RDM of any normalized symmetric $N$-quDit state $\psi$ like (7) can be expressed as

$$
\rho_{1}(\psi)=\frac{1}{N} \sum_{i, j=1}^{D}\left\langle S_{j i}\right\rangle E_{i j}
$$

with $D$-spin EVs (8). Note that $\operatorname{tr}\left[\rho_{1}(\psi)\right]=1$ since $\sum_{i=1}^{D}\left\langle S_{i i}\right\rangle=N$ (total population of the $D$ levels), which is related to the linear Casimir operator $C_{1}=\sum_{i=1}^{D} S_{i i}$ of $\mathrm{U}(D)$. From the condition $\operatorname{tr}\left(\rho_{1}(\psi)^{2}\right) \leq 1$, we obtain the general relation

$$
\sum_{i, j=1}^{D}\left|\left\langle S_{i j}\right\rangle\right|^{2} \leq N^{2}
$$

which could be seen as a measure of the fluctuations or departure from the linear $C_{1}$ and quadratic $C_{2}$ Casimir operators given by $C_{1}=N \mathbb{1}$ and 


$$
C_{2}=\sum_{i, j=1}^{D} S_{i j} S_{j i}=N(N+D-1) \mathbb{1}
$$

The quantum limit $N^{2}$ in (40) is attained for DSCSs. Indeed, for $|\psi\rangle=|\mathbf{z}\rangle$ in (9), the DSCS operator EVs were calculated in (12). Therefore, the purity of the corresponding one-quDit/atom RDM is simply [we denote interatom purity by $\mathscr{P}^{\mathrm{a}}$ to distinguish from the interlevel purity $\mathscr{P}^{\ell}$ discussed in the previous section]

$$
\mathscr{S}_{1}^{\mathrm{a}}(\mathbf{z})=\operatorname{tr}\left(\rho_{1}(\mathbf{z})^{2}\right)=\frac{1}{N^{2}} \sum_{i, j=1}^{D}\left|\left\langle\mathbf{z}\left|S_{i j}\right| \mathbf{z}\right\rangle\right|^{2}=\sum_{i, j=1}^{D} \frac{\left|z_{i}\right|^{2}\left|z_{j}\right|^{2}}{|\mathbf{z}|^{4}}=1,
$$

which means that there is not entanglement between atoms in a DSCS. This is because a DSCS is eventually obtained by rotating each atom individually. The situation changes when we deal with parity-adapted DSCSs or "Schrödinger cat states" (20). Indeed, the one-quDit RDM $\rho_{1}$ (DCAT) does not correspond now to a pure state since, using the $D$-spin EVs on a DCAT state (26), the purity gives

$$
\begin{aligned}
\mathscr{P}_{1}^{\mathrm{a}}(\text { DCAT }) & =\operatorname{tr}\left(\rho_{1}(\mathrm{DCAT})^{2}\right) \\
& =\frac{\left(\sum_{\mathfrak{b}}\left(\mathbf{z}^{\mathbb{b}} \cdot \mathbf{z}\right)^{N-1}\right)^{2}+\sum_{i=2}^{D}\left|z_{i}\right|^{4}\left(\sum_{\mathfrak{b}}(-1)^{b_{i}}\left(\mathbf{z}^{\mathbb{b}} \cdot \mathbf{z}\right)^{N-1}\right)^{2}}{\left(\sum_{\mathfrak{b}}\left(\mathbf{z}^{\mathfrak{b}} \cdot \mathbf{z}\right)^{N}\right)^{2}} \leq 1 .
\end{aligned}
$$

That is, unlike $|\mathbf{z}\rangle$, the Schrödinger cat $|\mathrm{DCAT}\rangle$ is not separable in the tensor product Hilbert space $\left[\mathbb{C}^{D}\right]^{\otimes N}$. In Fig. 3, we represent contour plots of linear and von Neumann

$$
\mathscr{L}_{1}^{\mathrm{a}}=\frac{D}{D-1}\left(1-\mathscr{P}_{1}^{\mathrm{a}}\right), \quad \delta_{1}^{\mathrm{a}}=-\operatorname{tr}\left(\rho_{1} \log _{D} \rho_{1}\right)
$$

entanglement entropies of the one-qutrit RDM $\rho_{1}$ (3CAT) of a U(3) Schrödinger cat (23) as a function of the phase-space $\mathbb{C} P^{2}$ coordinates $\alpha, \beta$ [actually, they just depend on the moduli]. Both entropies are again normalized to 1 . They attain their maximum value of 1 at the phase-space point $(\alpha, \beta)=(1,1)$ corresponding to a maximally mixed RDM. Figure 3 also shows (in magenta color) the stationary curve $(\alpha(\lambda), \beta(\lambda))$ previously mentioned at the end of Sect. 3.1 in relation to Fig. 2. For high interactions we have $(\alpha(\lambda), \beta(\lambda)) \stackrel{\lambda \rightarrow \infty}{\longrightarrow}(1,1)$, which means that highly coupled atoms are maximally entangled in a cat-like ground state (see later in Sects. 5 and 6 for more information).

To finish this section, let us comment on one-quDit entanglement for NODON states (30). Taking into account the $D$-spin EV (31), the one-quDit RDM of a NODON is simply $\rho_{1}($ NODON $)=\frac{1}{D} \mathbb{1}_{D}$ and its linear entropy $\mathscr{L}_{1}^{\mathrm{a}}=1$, implying maximally mixed RDM. 


\subsubsection{Two-quDit reduced density matrices}

Likewise, any density matrix of two quDits can be written as

$$
\rho_{2}=\sum_{i, j, k, l=1}^{D} r_{i j k l} E_{i j} \otimes E_{k l}, \quad r_{i j k l}=\operatorname{tr}\left(\rho_{2} E_{j i} \otimes E_{l k}\right)=\left\langle E_{j i} \otimes E_{l k}\right\rangle .
$$

with $\bar{r}_{i j k l}=r_{j i l k}$ complex parameters subject to $\operatorname{tr}\left[\rho_{2}\right]=1$ and $0<\operatorname{tr}\left[\left(\rho_{2}\right)^{2}\right] \leq 1$. Now we need to express the RDM on a pair of particles, extracted at random from a symmetric state of $N D$-level atoms, in terms of EVs of bilinear products of collective $D$-spin operators $S$. In particular, we have

$$
\begin{aligned}
\left\langle S_{i j} S_{k l}\right\rangle & =\sum_{\mu, \nu=1}^{N}\left\langle E_{i j}^{\mu} E_{k l}^{v}\right\rangle=\sum_{\mu=1}^{N} \delta_{j k}\left\langle E_{i l}^{\mu}\right\rangle+\sum_{\mu \neq \nu=1}^{N}\left\langle E_{i j}^{\mu} E_{k l}^{v}\right\rangle \\
& =\delta_{j k}\left\langle S_{i l}\right\rangle+N(N-1)\left\langle E_{i j}^{1} E_{k l}^{2}\right\rangle
\end{aligned}
$$

due to indistinguishableness. Therefore, the two-particle RDM of a symmetric state $\psi$ of $N>2$ quDits is written as

$$
\rho_{2}(\psi)=\frac{1}{N(N-1)} \sum_{i, j, k, l=1}^{D}\left(\left\langle S_{j i} S_{l k}\right\rangle-\delta_{i l}\left\langle S_{j k}\right\rangle\right) E_{i j} \otimes E_{k l}
$$

Using the Casimir values (41), one can directly prove that $\operatorname{tr}\left[\rho_{2}(\psi)\right]=1$ for any normalized symmetric state $\psi$. The case $D=2$ was considered by Wang and Mølmer in [11]. The procedure is straightforwardly extended to $\rho_{M}$ for an arbitrary number $M \leq N / 2$ of quDits. The purity of $\rho_{2}(\psi)$ can be compactly written as

$$
\mathscr{P}_{2}^{\mathrm{a}}(\psi)=\operatorname{tr}\left(\rho_{2}(\psi)^{2}\right)=\frac{1}{N^{2}(N-1)^{2}}\left[\sum_{i, j, k, l=1}^{D}\left\langle S_{j i} S_{l k}\right\rangle\left\langle S_{i j} S_{k l}\right\rangle-2 \sum_{i, j, k=1}^{D}\left\langle S_{j i} S_{k j}\right\rangle\left\langle S_{i k}\right\rangle+\sum_{i, j=1}^{D}\left\langle S_{i i}\right\rangle\left\langle S_{j j}\right\rangle\right] .
$$

In order to construct the two-particle RDM of a DSCS (9), we need the EVs of quadratic powers (14). With these ingredients, we can easily compute the two-particle RDM of a DSCS (9) which, for large $N$ has the following asymptotic expression

$$
\rho_{2}(\mathbf{z})=\sum_{i, j, k, l=1}^{D}\left(\bar{z}_{j} z_{i} \bar{z}_{l} z_{k}+O(1 / N)\right) E_{i j} \otimes E_{k l}
$$

The purity of $\rho_{2}(\mathbf{z})$ is 1 since $\mathbf{z}$ is separable in the tensor product Hilbert space $\left[\mathbb{C}^{D}\right]^{\otimes N}$, as we have already commented. Moreover, one can see that $\rho_{2}(\mathbf{z})=\rho_{1}(\mathbf{z}) \otimes \rho_{1}(\mathbf{z})$. However, the Schrödinger cat (20) is non-separable and has an intrinsic pairwise 

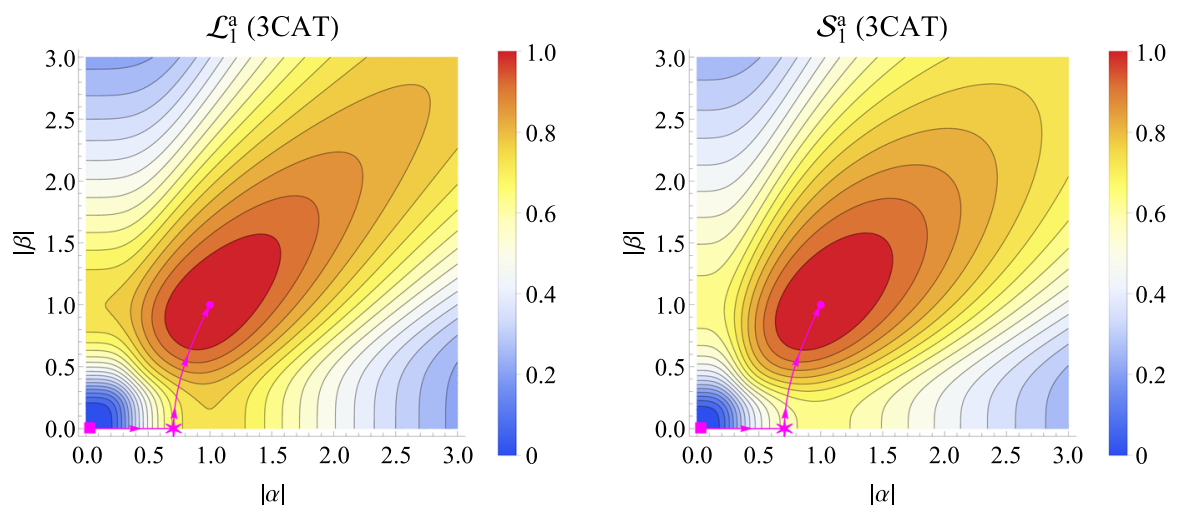

Fig. 3 DD plots of linear $\mathscr{L}_{1}^{\mathrm{a}}$ and von Neumann $\delta_{1}^{\mathrm{a}}$ entanglement entropies of the one-qutrit RDM $\rho_{1}$ (3CAT) of a U(3) Schrödinger cat (23) for $N=10$ atoms, as a function of the phase-space coordinates $\alpha, \beta$ (they just depend on moduli). The meaning of the magenta curve is the same as in Fig. 2
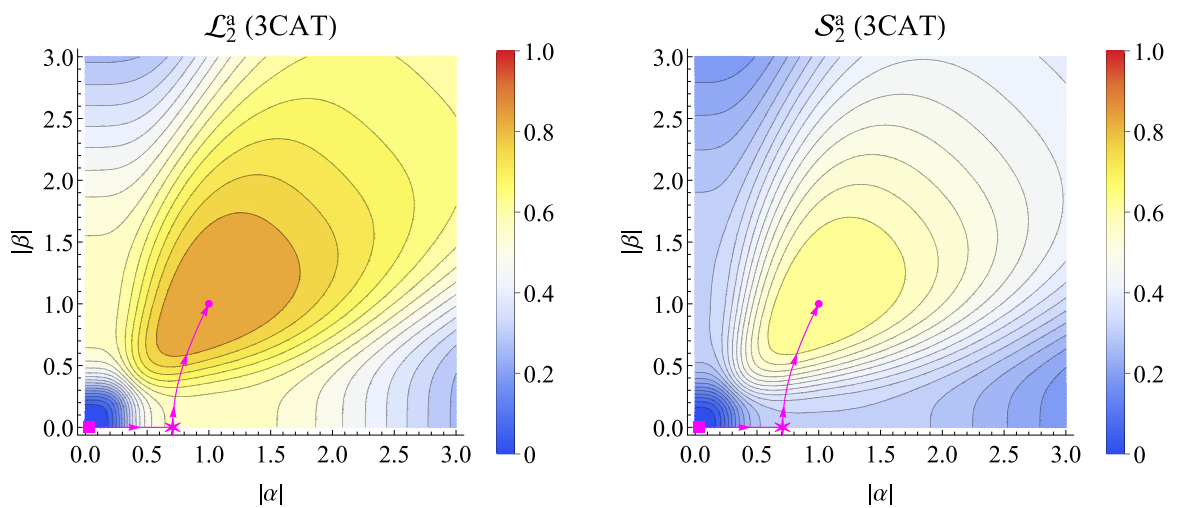

Fig. 4 Contour plots of linear $\mathscr{L}_{2}^{\mathrm{a}}$ and von Neumann $\delta_{2}^{\mathrm{a}}$ entanglement entropies of the two-qutrit RDM $\rho_{2}$ (3CAT) of a U(3) Schrödinger cat (23) for $N=10$ atoms, as a function of the phase-space coordinates $\alpha, \beta$ (they just depend on moduli). The meaning of the magenta curve is the same as in Fig. 2

entanglement. Taking into account the particular structure of linear (26) and quadratic (27) $D$-spin operator EVs, The general formula (48) becomes

$$
\mathscr{P}_{2}^{\mathrm{a}}(\mathrm{DCAT})=\frac{1}{N^{2}(N-1)^{2}}\left[\sum_{i, j, k, l=1}^{D}\left\langle S_{j i} S_{l k}\right\rangle\left\langle S_{i j} S_{k l}\right\rangle+\sum_{i, j=1}^{D}\left\langle S_{j i} S_{i j}\right\rangle\left(\left\langle S_{i j} S_{j i}\right\rangle-2\left\langle S_{i i}\right\rangle\right)+\sum_{i, j=1}^{D}\left\langle S_{i i}\right\rangle\left\langle S_{j j}\right\rangle\right] .
$$

In Fig. 4, we represent contour plots of normalized linear and von Neumann

$$
\mathscr{L}_{2}^{\mathrm{a}}=\frac{D^{2}}{D^{2}-1}\left(1-\mathscr{P}_{2}^{\mathrm{a}}\right), \quad \mathcal{S}_{2}^{\mathrm{a}}=-\operatorname{tr}\left(\rho_{2} \log _{D^{2}} \rho_{2}\right)
$$


entanglement entropies for the two-qutrit RDM $\rho_{2}$ (3CAT) of a U(3) Schrödinger cat (23) as a function of the phase-space $\mathbb{C} P^{2}$ coordinates $\alpha, \beta$ [they just depend on the moduli]. As for the one-quDit case, they attain their maximum value at the phase-space point $(\alpha, \beta)=(1,1)$; however, unlike the one-quDit case, pairwise entanglement entropies do not attain the maximum value of 1 at this point, but $\mathscr{L}_{2}^{\mathrm{a}}=5 / 6$ and $\mathcal{S}_{2}^{\mathrm{a}} \simeq$ 0.623 for large $N$. As already commented, variational (spin coherent) approximations to the ground state of the LMG three-level atom model [discussed later in Sect. 5] recover this maximum entanglement point $(\alpha, \beta)=(1,1)$ at high interactions $\lambda \rightarrow \infty$ (as can be seen in the magenta curve).

For NODON states (30), the two-quDit RDM is

$$
\rho_{2}(\mathrm{NODON})=\frac{1}{D} \sum_{k=1}^{D} E_{k k} \otimes E_{k k},
$$

and therefore $\rho_{2}(\mathrm{NODON})^{2}=\frac{1}{D} \rho_{2}$ (NODON), which means that the linear entropy is $\mathscr{L}_{2}^{\mathrm{a}}(\mathrm{NODON})=D /(D+1)$, indicating a high level of pairwise entanglement in a NODON state.

\section{SU(D)-spin squeezing: a proposal}

As we have already commented in the introduction, Wang and Sanders [22] showed a direct relation between the concurrence $C$, extracted from the two-qubit RDM (47) for $D=2$, and the $\mathrm{SU}(2)$ spin $\vec{J}=\left(J_{x}, J_{y}, J_{z}\right)$ squeezing parameter

$$
\begin{aligned}
\xi^{2} & =\frac{4}{N} \min _{\theta}\left\langle\left(\cos (\theta) J_{x}+\sin (\theta) J_{y}\right)^{2}\right\rangle \\
& =\frac{2}{N}\left[\left\langle J_{x}^{2}+J_{y}^{2}\right\rangle-\sqrt{\left\langle J_{x}^{2}-J_{y}^{2}\right\rangle^{2}+\left\langle J_{x} J_{y}+J_{y} J_{x}\right\rangle^{2}}\right]
\end{aligned}
$$

introduced by [15], which measures spin fluctuations in an orthogonal direction to the mean value $\langle\vec{J}\rangle$ with minimal variance. Actually, the definition (53) refers to even and odd symmetric multi-qubit states [remember the extension of this concept to multiquDits after (17)] for which $\langle\vec{J}\rangle=\left(0,0,\left\langle J_{z}\right\rangle\right)$ and therefore the orthogonal direction lies in the plane XY. This definition can be extended to even and odd symmetric multiquDit states for which $\left\langle S_{i j}\right\rangle \propto \delta_{i j}$ [see, e.g., (26) for the case of the even DCAT state]. Using the embedding (3) of $D(D-1) / 2 \mathrm{SU}(2)$ spin subalgebras into $\mathrm{U}(D)$, and mimicking (53), we can define $D(D-1) / 2$ spin squeezing parameters $\xi_{i j}, i>j$ for $D$-spin systems as:

$$
\xi_{i j}^{2}=\frac{1}{N(D-1)}\left[\left\langle S_{i j} S_{j i}+S_{j i} S_{i j}\right\rangle-2\left|\left\langle S_{i j}^{2}\right\rangle\right|\right], \quad i>j=1, \ldots, D-1
$$




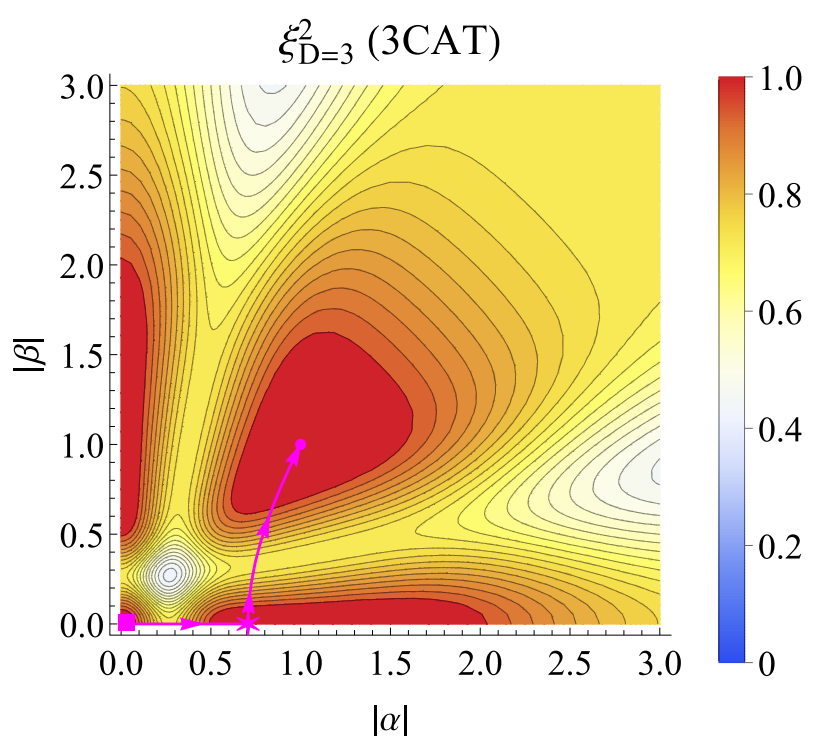

Fig. 5 Contour plots of the squeezing parameter $\xi_{D=3}^{2}$ of a $\mathrm{U}(3)$ Schrödinger cat for $N=10$ atoms, as a function of the phase-space coordinates $\alpha, \beta$ (it just depends on moduli). The meaning of the magenta curve is the same as in previous figures

We have chosen the normalization factor $\frac{1}{N(D-1)}$ so that (54) reduces to (53) for $D=2$ and so that the total $D$-spin squeezing parameter

$$
\xi_{D}^{2}=\sum_{i>j=1}^{D} \xi_{i j}^{2}
$$

is one (no squeezing) for the DSCSs $|\mathbf{z}\rangle$ in (9). Actually, for DSCSs we have that $\xi_{i j}^{2}=\left(\left|z_{i}\right|^{2}+\left|z_{j}\right|^{2}\right) /\left(|\mathbf{z}|^{2}(D-1)\right)$ is written in terms of average level populations $\left\langle\mathbf{z}\left|S_{i i}\right| \mathbf{z}\right\rangle=N\left|z_{i}\right|^{2} /|\mathbf{z}|^{2}$ of levels $i$ and $j$, according to (12). Therefore, the presence of $D$-spin squeezing means in general that $\xi_{D}^{2}<1$. Using the EVs (31) for NODON states (30), the corresponding spin squeezing parameters are $\xi_{i j}=2 /[D(D-1)]$, which gives $\xi_{D}^{2}=1$, thus implying that NODON states do not exhibit spin squeezing.

Note that $D$-spin squeezing parameters $\xi_{i j}$ are constructed in terms of $D$-spin quadratic EVs, as the two-quDit RDM (47) and its purity (48) do. Therefore, the deep relation between pairwise entanglement and spin squeezing revealed by Wang-Sanders in [22] for symmetric multi-qubit systems is extensible to symmetric multi-quDits in the sense proposed here. In Fig. 5, we show a contour plot of the total $D$-spin squeezing parameter $\xi_{D}^{2}$ for the 3CAT. As in previous figures, the magenta curve represents the trajectory in phase space of the stationary points (59) of the energy surface (57) of the three-level LMG Hamiltonian (56) as a function of the control parameter $\lambda$. See later around Fig. 9 in Sect. 6 for further discussion. 


\section{LMG model for three-level atoms and its quantum phase diagram}

In this section, we apply the previous mathematical machinery to the study and characterization of the phase diagram of quantum critical $D$-level Lipkin-Meshkov-Glick atom models. The standard case of $D=2$ level atoms has already been studied in the literature (see, e.g., [37]). We shall restrict ourselves to $D=3$ level atoms for practical calculations, although the procedure can be easily extended to general $D$. In particular, we propose the following LMG-type Hamiltonian

$$
H=\frac{\epsilon}{N}\left(S_{33}-S_{11}\right)-\frac{\lambda}{N(N-1)} \sum_{i \neq j=1}^{3} S_{i j}^{2},
$$

written in terms of collective U(3)-spin operators $S_{i j}$. Hamiltonians of this kind have already been proposed in the literature [38-42] (see also [21] for the role of mixed symmetry sectors in QPTs of multi-quDit LMG systems). We place levels symmetrically about $i=2$, with intensive energy splitting per particle $\epsilon / N$. For simplicity, we consider equal interactions, with coupling constant $\lambda$, for atoms in different levels, and vanishing interactions for atoms in the same level (i.e., we discard interactions of the form $S_{i j} S_{j i}$ ). Therefore, $H$ is invariant under parity transformations $\Pi_{j}$ in (17), since the interaction term scatters pairs of particles conserving the parity of the population $n_{j}$ in each level $j=1, \ldots, D$. Energy levels have good parity, the ground state being an even state. We divide the two-body interaction in (56) by the number of atom pairs $N(N-1)$ to make $H$ an intensive quantity, since we are interested in the thermodynamic limit $N \rightarrow \infty$. We shall see that parity symmetry is spontaneously broken in this limit.

As already pointed long ago by Gilmore and coworkers [14,55], coherent states constitute in general a powerful tool for rigorously studying the ground state and thermodynamic critical properties of some physical systems. The energy surface associated with a Hamiltonian density $H$ is defined in general as the coherent state expectation value of the Hamiltonian density in the thermodynamic limit. In our case, the energy surface acquires the following form

$$
\begin{aligned}
E_{(\alpha, \beta)}(\epsilon, \lambda)= & \lim _{N \rightarrow \infty}\langle\mathbf{z}|H| \mathbf{z}\rangle=\epsilon \frac{\beta \bar{\beta}-1}{\alpha \bar{\alpha}+\beta \bar{\beta}+1} \\
& -\lambda \frac{\alpha^{2}\left(\bar{\beta}^{2}+1\right)+\left(\beta^{2}+1\right) \bar{\alpha}^{2}+\bar{\beta}^{2}+\beta^{2}}{(\alpha \bar{\alpha}+\beta \bar{\beta}+1)^{2}},
\end{aligned}
$$

where we have used DSCS EVs of linear (12) and quadratic (14) powers of $D$-spin operators $S_{i j}$ [actually, linear powers are enough due to the lack of quantum spin fluctuations in the thermodynamic limit (16)], and we have used the parametrization $\mathbf{z}=(1, \alpha, \beta)$, as in Eq. (23), for U(3) SCSs. Note that this energy surface is invariant under $\alpha \rightarrow-\alpha$ and $\beta \rightarrow-\beta$, which is a consequence of the inherent parity symmetry of the Hamiltonian (56). 
The minimum energy

$$
E_{0}(\epsilon, \lambda)=\min _{\alpha, \beta \in \mathbb{C}} E_{(\alpha, \beta)}(\epsilon, \lambda)
$$

is attained at the stationary (real) phase-space values $\alpha_{0}^{ \pm}= \pm \alpha_{0}$ and $\beta_{0}^{ \pm}= \pm \beta_{0}$ with

$$
\begin{aligned}
& \alpha_{0}(\epsilon, \lambda)= \begin{cases}0, & 0 \leq \lambda \leq \frac{\epsilon}{2}, \\
\sqrt{\frac{2 \lambda-\epsilon}{2 \lambda+\epsilon}}, & \frac{\epsilon}{2} \leq \lambda \leq \frac{3 \epsilon}{2}, \\
\sqrt{\frac{2 \lambda}{2 \lambda+3 \epsilon}}, & \lambda \geq \frac{3 \epsilon}{2},\end{cases} \\
& \beta_{0}(\epsilon, \lambda)= \begin{cases}0, & 0 \leq \lambda \leq \frac{3 \epsilon}{2}, \\
\sqrt{\frac{2 \lambda-3 \epsilon}{2 \lambda+3 \epsilon}}, & \lambda \geq \frac{3 \epsilon}{2} .\end{cases}
\end{aligned}
$$

In Figs. 2, 3, 4 and 5, we plotted (in magenta color) the stationary-point curve $\left(\alpha_{0}(\lambda), \beta_{0}(\lambda)\right)$ on top of level, one- and two-qutrit entanglement entropies and squeezing parameter, noting that $\left(\alpha_{0}(\lambda), \beta_{0}(\lambda)\right) \rightarrow(1,1)$ for high $\lambda \rightarrow \infty$ interactions. We will come to this later in Sect. 6. Inserting (59) into (57) gives the ground state energy density at the thermodynamic limit

$$
E_{0}(\epsilon, \lambda)= \begin{cases}-\epsilon, & 0 \leq \lambda \leq \frac{\epsilon}{2} \\ -\frac{(2 \lambda+\epsilon)^{2}}{8 \lambda}, & \frac{\epsilon}{2} \leq \lambda \leq \frac{3 \epsilon}{2} \\ -\frac{4 \lambda^{2}+3 \epsilon^{2}}{6 \lambda}, & \lambda \geq \frac{3 \epsilon}{2}\end{cases}
$$

Here, we clearly distinguish three different phases: I, II and III, and two second-order QPTs at $\lambda_{\mathrm{I} \leftrightarrow \mathrm{II}}^{(0)}=\epsilon / 2$ and $\lambda_{\mathrm{II} \leftrightarrow \mathrm{III}}^{(0)}=3 \epsilon / 2$, respectively, where $\frac{\partial^{2} E_{0}(\epsilon, \lambda)}{\partial \lambda^{2}}$ are discontinuous. In the stationary (magenta) curve $\left(\alpha_{0}(\lambda), \beta_{0}(\lambda)\right)$, the phase I corresponds to the origin $\left(\alpha_{0}, \beta_{0}\right)=(0,0)$ (squared point), phase II corresponds to the horizontal part $\beta_{0}=0$ up to the star point, and phase III corresponds to $\beta_{0} \neq 0$.

Note that the ground state is fourfold degenerated in the thermodynamic limit since the four U(3) SCSs $\left|\mathbf{z}_{0}^{ \pm \pm}\right\rangle=\left|1, \pm \alpha_{0}, \pm \beta_{0}\right\rangle$ have the same energy density $E_{0}$. These four U(3) SCSs are related by parity transformations $\Pi_{j}$ in (17) and, therefore, parity symmetry is spontaneously broken in the thermodynamic limit. In order to have good variational states for finite $N$, to compare with numerical calculations, we have two possibilities: 1) either we use the 3CAT (23) as an ansatz for the ground state, minimizing $\langle 3 \mathrm{CAT}|H| 3 \mathrm{CAT}\rangle$, or 2 ) we restore the parity symmetry of the $\mathrm{U}(3)$ SCS $\left|1, \alpha_{0}, \beta_{0}\right\rangle$ for finite $N$ by projecting on the even parity sector. Although the first possibility offers a more accurate variational approximation to the ground state, it entails a more tedious numerical minimization than the one already obtained in (58) for $N \rightarrow \infty$. Therefore, we shall use the second possibility which, despite being less accurate, it is straightforward and good enough for our purposes. That is, we shall use the 3CAT (23), evaluated at $\alpha=\alpha_{0}$ and $\beta=\beta_{0}$ and conveniently normalized (24), as a variational approximation $\left|3 \mathrm{CAT}_{0}\right\rangle$ to the numerical (exact) ground state $\left|\psi_{0}\right\rangle$ for finite $N$. 

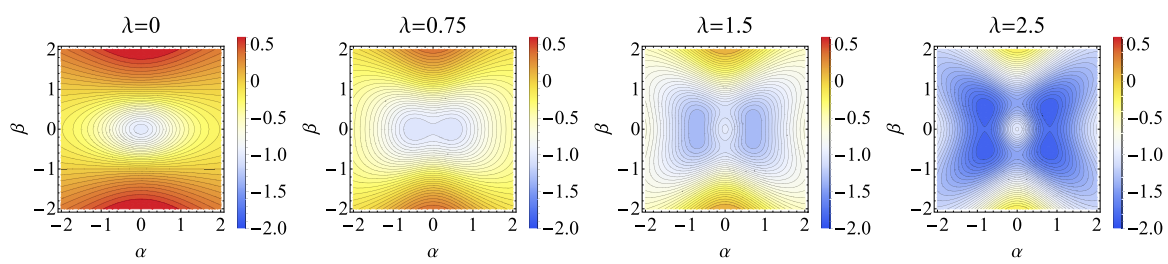

Fig. 6 Contour plot of the energy surface (57) for real $\alpha$ and $\beta$, in the vicinity of the critical points $\lambda=1 / 2$ and $\lambda=3 / 2$ (in $\epsilon$ units). Degenerate minima are perceived in phases II $\left(\frac{1}{2} \leq \lambda \leq \frac{3}{2}\right)$ and III $\left(\lambda \geq \frac{3}{2}\right)$

\section{Entanglement and squeezing as signatures of QPTs}

The objective in this section is to use level and particle entanglement and squeezing measures as signatures of QPTs in these LMG models, playing the role of order parameters that characterize the different phases or markers of the corresponding critical points. We restrict ourselves to linear entropy which, as already shown, gives qualitative information similar to von Neumann entropy for this study, with the advantage that it requires less computational resources. As already commented, Refs. [44,45] contain more general information about the relation between both entropies. Linear entropies of one- and two-qutrit RDMs turn also to provide similar qualitative information, although pairwise entanglement shows a more direct relation to spin squeezing.

We have numerically diagonalized the Hamiltonian (56) for $N=50$ three-level atoms, and several values of $\lambda$ (in $\epsilon$ units), and we have calculated level, one- and twoqutrit entanglement linear entropies for the ground state $\left|\psi_{0}\right\rangle=\sum_{\vec{n}}^{\prime} c_{\vec{n}}|\vec{n}\rangle$, plugging the coefficients $c_{\vec{n}}$ into $(8,33,48)$. We have also calculated level and atom entanglement linear entropies for the variational approximation $\left|3 \mathrm{CAT}_{0}\right\rangle$ to the ground state $\left|\psi_{0}\right\rangle$ discussed in the previous section for $N=50$. In Fig. 7, we compare numerical with variational ground state entanglement measures between levels $i=1,2,3$. According to Fig. 7, we see that, in phase I, $0 \leq \lambda \leq 1 / 2$, variational results indicate that there is no entanglement between levels, whereas numerical results show a small (but nonzero) entanglement for $N=50$. In phase II, $1 / 2 \leq \lambda \leq 3 / 2$, levels $i=1$ and $i=2$ get entangled, but level $i=3$ remains almost disconnected. In phase III, $\lambda \geq 3 / 2$, level $i=3$ gets entangled too. Interlevel entanglement grows with $\lambda$ attaining the maximum value of 0.84 at the limiting point $\left(\alpha_{0}(\infty), \beta_{0}(\infty)\right)=(1,1)$ for $N=50$. This behavior of the interlevel entropy for the 3CAT variational state can be also appreciated by looking at the stationary (magenta) curve in Fig. 2 with relation to the isentropic curves.

Concerning atom entanglement, Fig. 8 shows a better agreement between variational and numerical results, showing a rise of entanglement when the coupling strength $\lambda$ grows across the three phases, attaining values close to the large $N$ maximum values $\mathscr{L}_{1}^{\mathrm{a}}=1$ and $\mathscr{L}_{2}^{\mathrm{a}}=5 / 6$ at the limiting point $\left(\alpha_{0}(\infty), \beta_{0}(\infty)\right) \rightarrow(1,1)$. The entanglement growth is more abrupt between phases I and II than between phases II and III. We see that both, level and atom entanglement measures capture differences between the three phases, even for finite $N$, and therefore they can be considered as precursors of the corresponding QPT. The main features of the inter-atom entanglement entropy for the 3CAT variational state are also captured by the trajectory of the stationary (magenta) curve in Figs. 3 and 4 through the isentropic curves. 


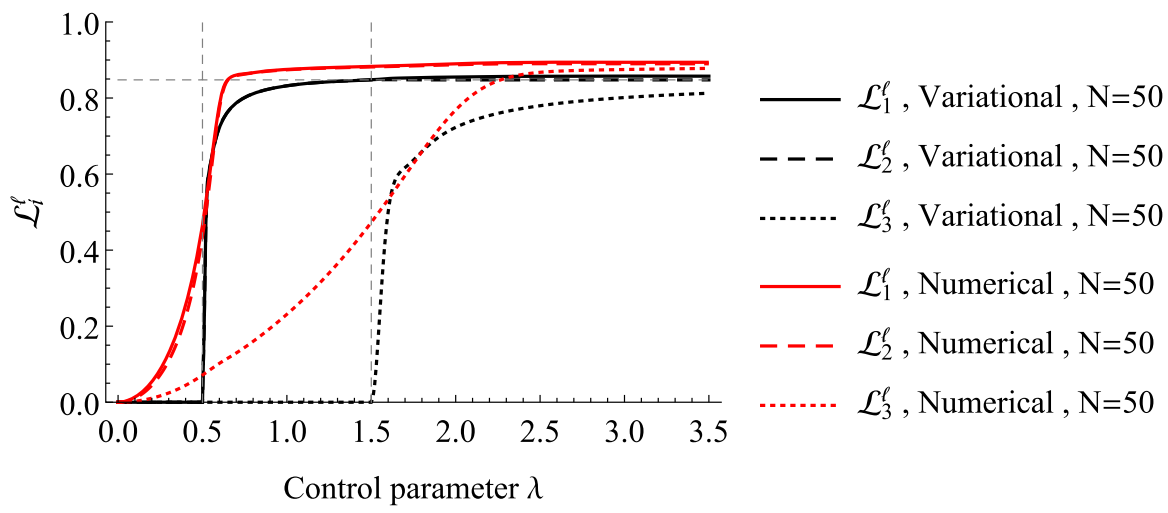

Fig. 7 Level entanglement linear entropies $\mathscr{L}_{i}^{\ell}$ (for levels $i=1,2$ and 3 ) of the ground state of the threelevel atom LMG model Hamiltonian (56), for $N=50$ atoms, as a function of the control parameter $\lambda$ (in $\epsilon$ units). Critical points, at which a QPT takes place, are marked with vertical grid lines, whereas the horizontal grid line labels the asymptotic value $\mathscr{L}_{i}^{\ell} \rightarrow 1-2 / \sqrt{\pi N} \simeq 0.84$ of the entropies. We compare exact results, obtained from numerical diagonalization of the Hamiltonian, with variational (analytical) results obtained from a parity symmetry restoration (in terms of Schrödinger cats) of mean-field results

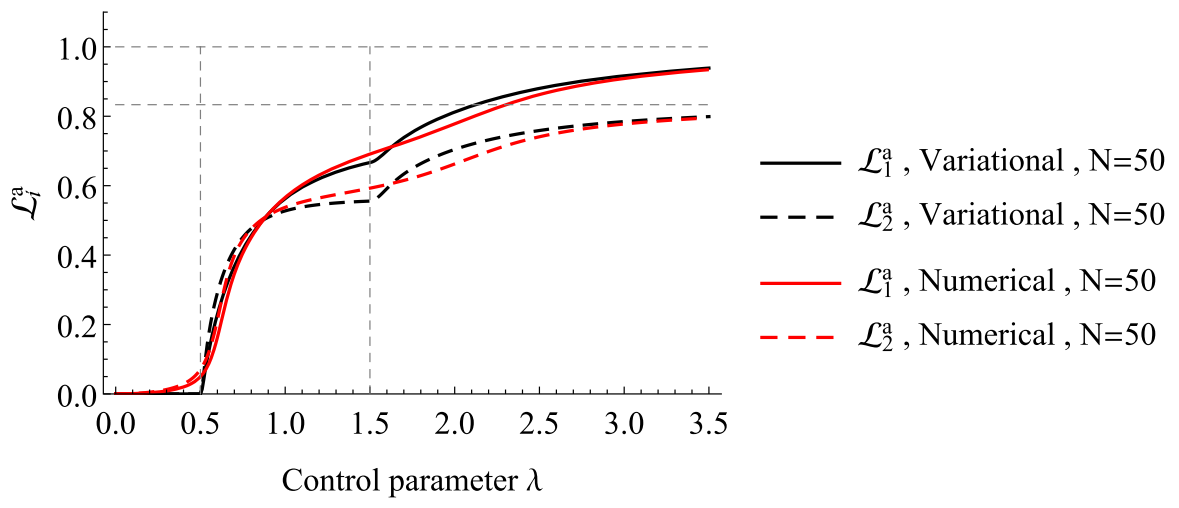

Fig. 8 One-qutrit $\mathscr{L}_{1}^{\mathrm{a}}$ and two-qutrit $\mathscr{L}_{2}^{\mathrm{a}}$ entanglement linear entropies of the ground state of the three-level atom LMG model Hamiltonian (56) as a function of the control parameter $\lambda$ (in $\epsilon$ units). Critical points, at which a QPT takes place, are marked with vertical grid lines, whereas horizontal grid lines label the asymptotic values $\mathscr{L}_{1}^{\mathrm{a}} \rightarrow 1$ and $\mathscr{L}_{2}^{\mathrm{a}} \rightarrow 5 / 6$ of the entropies. We compare numerical with variational results for $N=50$ atoms

In Fig. 9, we represent the $D=3$ spin total squeezing parameter $\xi_{D}^{2}$ (55) of the variational and numerical ground states for $N=50$ atoms, as a function of the control parameter $\lambda$ (in $\epsilon$ units). The results reveal a clear growth of squeezing at the critical points, the change being more abrupt at these points for the variational (parity-adapted mean field) than for the numerical ground state. Note that the variational ground state only shows squeezing $\left(\xi_{D}^{2}<1\right)$ at the critical points, whereas the numerical ground state exhibits squeezing for any $\lambda \neq 0$. Looking at the stationary (magenta) curve of Fig. 5, we appreciate that it practically lies in regions of no squeezing (in red color) except near the critical points, where squeezing suddenly increases (yellow color regions). 


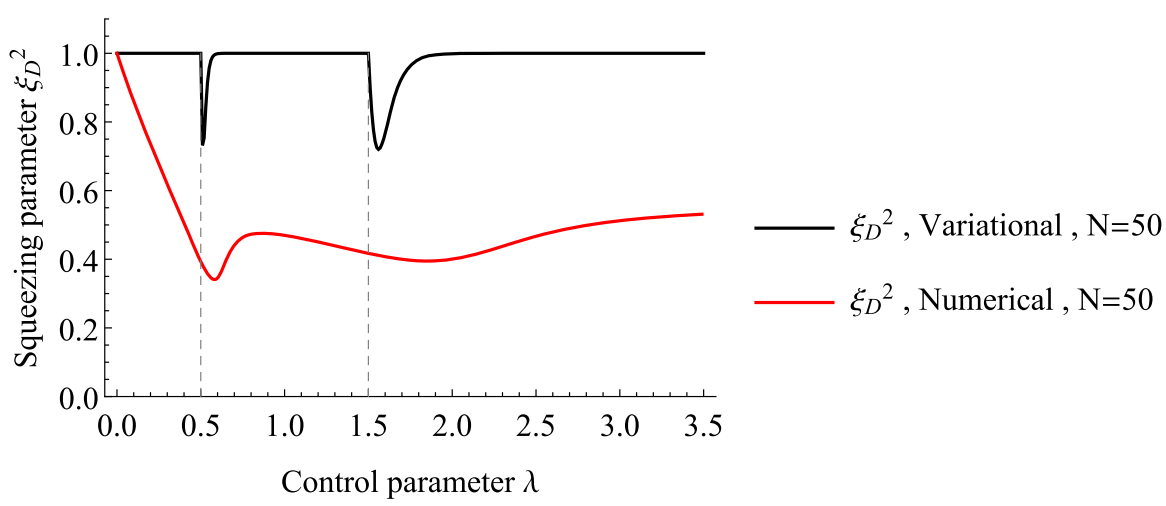

Fig. $9 D$-spin total squeezing parameter $\xi_{D}^{2}(55)$ of the ground state of the three-level atom LMG model Hamiltonian (56) as a function of the control parameter $\lambda$ (in $\epsilon$ units). Critical points, at which a QPT takes place, are marked with vertical grid lines. We compare numerical with variational results for $N=50$ atoms

\section{Conclusions and outlook}

We have extended the concept of pairwise entanglement and spin squeezing for symmetric multi-qubits (namely identical two-level atoms) to general symmetric multi-quDits (namely identical $D$-level atoms). For it, we have firstly computed expectation values of $\mathrm{U}(D)$ spin operators $S_{i j}$ in general symmetric multi-quDit states like: $\mathrm{U}(D)$-spin coherent states, their adaptation to parity (Schrödinger DCAT states), and an extension of NOON states to $D$ levels (NODON states). The reduced density matrices to one and two quDits extracted at random from a symmetric multi-quDit state exhibit atom entanglement for DCAT states, but not for $\mathrm{U}(D)$-spin coherent states. We have used entanglement to characterize quantum phase transitions of LMG $D$-level atom models (we have restricted to $D=3$ for simplicity), where DCAT states (as an adaptation to parity of mean-field spin coherent states) turn out to be a reasonable good variational approximation to the exact (numerical) ground state. We have also proposed an extension of standard SU(2)-spin squeezing to $\mathrm{SU}(D)$-spin operators, which recovers $D=2$ as a particular case. We have evaluated $\mathrm{SU}(3)$-spin squeezing of the ground state of the LMG three-level atom model, as a function of the control parameter $\lambda$, and we have seen that squeezing grows in the neighborhood of critical points $\lambda_{c}$, therefore serving as a marker of the corresponding quantum phase transition. A deeper study and discussion of squeezing in these models requires a phase-space approach in terms of a coherent (Bargmann) representation of states, such as the Husimi and Wigner functions, and it will be the subject of the future work.

Acknowledgements We thank the support of the Spanish MICINN through the Project PGC2018-097831B-I00 and Junta de Andalucía through the Projects SOMM17/6105/UGR, UHU-1262561 and FQM-381. JG also thanks MICINN for financial support from FIS2017-84440-C2-2-P. AM thanks the Spanish MIU for the FPU19/06376 predoctoral fellowship. We all thank Octavio Castaños for his valuable collaboration in the early stages of this work.

Funding Open Access funding provided thanks to the CRUE-CSIC agreement with Springer Nature. 
Open Access This article is licensed under a Creative Commons Attribution 4.0 International License, which permits use, sharing, adaptation, distribution and reproduction in any medium or format, as long as you give appropriate credit to the original author(s) and the source, provide a link to the Creative Commons licence, and indicate if changes were made. The images or other third party material in this article are included in the article's Creative Commons licence, unless indicated otherwise in a credit line to the material. If material is not included in the article's Creative Commons licence and your intended use is not permitted by statutory regulation or exceeds the permitted use, you will need to obtain permission directly from the copyright holder. To view a copy of this licence, visit http://creativecommons.org/licenses/by/4.0/.

\section{References}

1. Amico, L., Fazio, R., Osterloh, A., Vedral, V.: Entanglement in many-body systems. Rev. Mod. Phys. 80, 517-576 (2008). https://doi.org/10.1103/RevModPhys.80.517

2. Pezzè, L., Smerzi, A., Oberthaler, M.K., Schmied, R., Treutlein, P.: Quantum metrology with nonclassical states of atomic ensembles. Rev. Mod. Phys. 90, 035005 (2018). https://doi.org/10.1103/ RevModPhys.90.035005

3. Horodecki, R., Horodecki, P., Horodecki, M., Horodecki, K.: Quantum entanglement. Rev. Mod. Phys. 81, 865-942 (2009). https://doi.org/10.1103/RevModPhys.81.865

4. Ma, J., Wang, X., Sun, C.P., Nori, F.: Quantum spin squeezing. Phys. Rep. 509, 89-165 (2011). https:// doi.org/10.1016/j.physrep.2011.08.003

5. Benatti, F., Floreanini, R., Franchini, F., Marzolino, U.: Entanglement in indistinguishable particle systems. Phys. Rep. 878, 1-27 (2020). https://doi.org/10.1016/j.physrep.2020.07.003

6. Morris, B., Yadin, B., Fadel, M., Zibold, T., Treutlein, P., Adesso, G.: Entanglement between identical particles is a useful and consistent resource. Phys. Rev. X 10, 041012 (2020). https://doi.org/10.1103/ PhysRevX.10.041012

7. Killoran, N., Cramer, M., Plenio, M.B.: Extracting entanglement from identical particles. Phys. Rev. Lett. 112, 150501 (2014). https://doi.org/10.1103/PhysRevLett.112.150501

8. Dalton, B.J., Goold, J., Garraway, B.M., Reid, M.D.: Quantum entanglement for systems of identical bosons: I. General features. Phys. Scripta 92, 023004 (2017a). https://doi.org/10.1088/1402-4896/92/ $2 / 023004$

9. Dalton, B.J., Goold, J., Garraway, B.M., Reid, M.D.: Quantum entanglement for systems of identical bosons: II. Spin squeezing and other entanglement tests. Phys. Scripta 92, 023005 (2017b). https:// doi.org/10.1088/1402-4896/92/2/023005

10. Dür, W., Vidal, G., Cirac, J.I.: Three qubits can be entangled in two inequivalent ways. Phys. Rev. A 62, 062314 (2000). https://doi.org/10.1103/PhysRevA.62.062314

11. Wang, X., Mølmer, K.: Pairwise entanglement in symmetric multi-qubit systems. Eur. Phys. J. D 18, 385-391 (2002). https://doi.org/10.1140/epjd/e20020045

12. Wootters, W.K.: Entanglement of formation of an arbitrary state of two qubits. Phys. Rev. Lett. 80, 2245-2248 (1998). https://doi.org/10.1103/PhysRevLett.80.2245

13. Radcliffe, J.M.: Some properties of coherent spin states. J. Phys. A Gen. Phys. 4, 313-323 (1971). https://doi.org/10.1088/0305-4470/4/3/009

14. Arecchi, F.T., Courtens, E., Gilmore, R., Thomas, H.: Atomic coherent states in quantum optics. Phys. Rev. A 6, 2211-2237 (1972). https://doi.org/10.1103/PhysRevA.6.2211

15. Kitagawa, M., Ueda, M.: Squeezed spin states. Phys. Rev. A 47, 5138-5143 (1993). https://doi.org/ 10.1103/PhysRevA.47.5138

16. Dodonov, V.V., Malkin, I.A., Manko, V.I.: Even and odd coherent states and excitations of a singular oscillator. Physica 72, 597-615 (1974). https://doi.org/10.1016/0031-8914(74)90215-8

17. Castaños, O., López-Peña, R., Man'ko, V.I.: Crystallized Schrödinger cat states. J. Russ. Laser Res. 16, 477-525 (1995). https://doi.org/10.1007/BF02581033

18. Nieto, M.M., Truax, D.R.: Squeezed states for general systems. Phys. Rev. Lett. 71, 2843-2846 (1993). https://doi.org/10.1103/PhysRevLett.71.2843

19. Bužek, V., Vidiella-Barranco, A., Knight, P.L.: Superpositions of coherent states: squeezing and dissipation. Phys. Rev. A 45, 6570-6585 (1992). https://doi.org/10.1103/PhysRevA.45.6570

20. Hillery, M.: Amplitude-squared squeezing of the electromagnetic field. Phys. Rev. A 36, 3796-3802 (1987). https://doi.org/10.1103/PhysRevA.36.3796 
21. Calixto, M., Mayorgas, A., Guerrero, J.: Role of mixed permutation symmetry sectors in the thermodynamic limit of critical three-level Lipkin-Meshkov-Glick atom models. Phys. Rev. E 103, 012116 (2021). https://doi.org/10.1103/PhysRevE.103.012116

22. Wang, X., Sanders, B.C.: Spin squeezing and pairwise entanglement for symmetric multiqubit states. Phys. Rev. A 68, 012101 (2003). https://doi.org/10.1103/PhysRevA.68.012101

23. Sørensen, A., Duan, L.M., Cirac, J.I., Zoller, P.: Many-particle entanglement with Bose-Einstein condensates. Nature 409, 63-66 (2001). https://doi.org/10.1038/35051038

24. Sørensen, A.S., Mølmer, K.: Entanglement and extreme spin squeezing. Phys. Rev. Lett. 86, 4431-4434 (2001). https://doi.org/10.1103/PhysRevLett.86.4431

25. Mølmer, K., Sørensen, A.: Multiparticle entanglement of hot trapped ions. Phys. Rev. Lett. 82, 18351838 (1999). https://doi.org/10.1103/PhysRevLett.82.1835

26. Sackett, C.A., Kielpinski, D., King, B.E., Langer, C., Meyer, V., Myatt, C.J., Rowe, M., Turchette, Q.A., Itano, W.M., Wineland, D.J., Monroe, C.: Experimental entanglement of four particles. Nature 404, 256-259 (2000). https://doi.org/10.1038/35005011

27. Castaños, O., Nahmad-Achar, E., López-Peña, R., Hirsch, J.G.: Superradiant phase in field-matter interactions. Phys. Rev. A 84, 013819 (2011). https://doi.org/10.1103/PhysRevA.84.013819

28. Romera, E., del Real, R., Calixto, M.: Husimi distribution and phase-space analysis of a Dicke-model quantum phase transition. Phys. Rev. A 85, 053831 (2012). https://doi.org/10.1103/PhysRevA.85. 053831

29. Cordero, S., Nahmad-Achar, E., López-Peña, R., Castaños, O.: Polychromatic phase diagram for $n$ level atoms interacting with $\ell$ modes of an electromagnetic field. Phys. Rev. A 92, 053843 (2015). https://doi.org/10.1103/PhysRevA.92.053843

30. López-Peña, R., Cordero, S., Nahmad-Achar, E., Castaños, O.: Symmetry adapted coherent states for three-level atoms interacting with one-mode radiation. Phys. Scr. 90, 068016 (2015). https://doi.org/ 10.1088/0031-8949/90/6/068016

31. Pérez-Campos, C., González-Alonso, J.R., Castaños, O., López-Peña, R.: Entanglement and localization of a two-mode Bose-Einstein condensate. Ann. Phys. 325, 325-344 (2010)

32. Calixto, M., Romera, E., del Real, R.: Parity-symmetry-adapted coherent states and entanglement in quantum phase transitions of vibron models. J. Phys. A Math. Theor. 45, 365301 (2012). https://doi. org/10.1088/1751-8113/45/36/365301

33. Calixto, M., Pérez-Bernal, F.: Entanglement in shape phase transitions of coupled molecular benders. Phys. Rev. A 89, 032126 (2014). https://doi.org/10.1103/PhysRevA.89.032126

34. Calixto, M., Peón-Nieto, C.: Husimi function and phase-space analysis of bilayer quantum hall systems at $v=\lambda$. J. Stat. Mech. Theory Exp. 2018, 053112 (2018). https://doi.org/10.1088/1742-5468/aabfcb

35. Castaños, O., López-Peña, R., Hirsch, J.G., López-Moreno, E.: Classical and quantum phase transitions in the Lipkin-Meshkov-Glick model. Phys. Rev. B 74, 104118 (2006). https://doi.org/10.1103/ PhysRevB.74.104118

36. Romera, E., Calixto, M., Castaños, O.: Phase space analysis of first-, second- and third-order quantum phase transitions in the Lipkin-Meshkov-Glick model. Phys. Scr. 89, 095103 (2014). https://doi.org/ 10.1088/0031-8949/89/9/095103

37. Calixto, M., Castaños, O., Romera, E.: Entanglement and quantum phase diagrams of symmetric multiqubit systems. J. Stat. Mech. Theory Exp. 2017, 103103 (2017). https://doi.org/10.1088/1742-5468/ aa8703

38. Gnutzmann, S., Kuś, M.: Coherent states and the classical limit on irreducible su3 representations. J. Phys. A Math. Gen. 31, 9871 (1999). https://doi.org/10.1088/0305-4470/31/49/011

39. Gnutzmann, S., Haake, F., Kuś, M.: Quantum chaos of su3 observables. J. Phys. A Math. Gen. 33, 143 (1999). https://doi.org/10.1088/0305-4470/33/1/309

40. Meredith, D.C., Koonin, S.E., Zirnbauer, M.R.: Quantum chaos in a schematic shell model. Phys. Rev. A 37, 3499-3513 (1988). https://doi.org/10.1103/PhysRevA.37.3499

41. Wang, W.-G., Izrailev, F.M., Casati, G.: Structure of eigenstates and local spectral density of states: a three-orbital schematic shell model. Phys. Rev. E 57, 323-339 (1998). https://doi.org/10.1103/ PhysRevE.57.323

42. Leboeuf, P., Saraceno, M.: Eigenfunctions of non-integrable systems in generalised phase spaces. J. Phys. A Math. Gen. 23, 1745 (1999). https://doi.org/10.1088/0305-4470/23/10/016

43. Iachello, F., Arima, A.: The Interacting Boson Model, Cambridge Monographs on Mathematical Physics. Cambridge University Press, Cambridge (1987). https://doi.org/10.1017/ CBO9780511895517 
44. Berry, D.W., Sanders, B.: Bounds on general entropy measures. J. Phys. A Math. Gen. 36, 12255 (2003). https://doi.org/10.1088/0305-4470/36/49/008

45. Wei, T.-C., Nemoto, K., Goldbart, P.M., Kwiat, P.G., Munro, W.J., Verstraete, F.: Maximal entanglement versus entropy for mixed quantum states. Phys. Rev. A 67, 022110 (2003). https://doi.org/10.1103/ PhysRevA.67.022110

46. Moshinsky, M.: The harmonic oscillator and supermultiplet theory: (i) the single shell picture. Nucl. Phys. 31, 384-405 (1962). https://doi.org/10.1016/0029-5582(62)90758-7

47. Moshinsky, M., Nagel, J.G.: Complete classification of states of supermultiplet theory. Phys. Lett. 5, 173-174 (1963). https://doi.org/10.1016/S0375-9601(63)92662-8

48. Biedenharn, L.C., Louck, J.D.: Angular Momentum in Quantum Physics: Theory and Application, Encyclopedia of Mathematics and Its Applications. Cambridge University Press, Cambridge (1984). https://doi.org/10.1017/CBO9780511759888

49. Humphreys, P.C., Barbieri, M., Datta, A., Walmsley, I.A.: Quantum enhanced multiple phase estimation. Phys. Rev. Lett. 111, 070403 (2013). https://doi.org/10.1103/PhysRevLett.111.070403

50. Zhang, L., Chan, K.W.C.: Scalable generation of multi-mode noon states for quantum multiple-phase estimation. Sci. Rep. 8, 11440 (2018). https://doi.org/10.1038/s41598-018-29828-2

51. Iachello, F., Levine, R.D.: Algebraic Theory of Molecules. Oxford University Press, Oxford (1995). https://doi.org/10.1002/bbpc.19950990427

52. Frank, A., Van Isacker, P.: Algebraic Methods in Molecular and Nuclear Structure Physics. Wiley, New York (1994)

53. Pérez-Bernal, F., Iachello, F.: Algebraic approach to two-dimensional systems: shape phase transitions, monodromy, and thermodynamic quantities. Phys. Rev. A 77, 032115 (2008). https://doi.org/10.1103/ PhysRevA.77.032115

54. Pérez-Fernández, P., Arias, J.M., García-Ramos, J.E., Pérez-Bernal, F.: Finite-size corrections in the bosonic algebraic approach to two-dimensional systems. Phys. Rev. A 83, 062125 (2011). https://doi. org/10.1103/PhysRevA.83.062125

55. Zhang, W.-M., Feng, D.H., Gilmore, R.: Coherent states: theory and some applications. Rev. Mod. Phys. 62, 867-927 (1990). https://doi.org/10.1103/RevModPhys.62.867

Publisher's Note Springer Nature remains neutral with regard to jurisdictional claims in published maps and institutional affiliations. 